\title{
Valuations on algebras with involution
}

\author{
J.-P. Tignol • A. R. Wadsworth
}

Received: 30 January 2009 / Revised: 16 July 2010 / Published online: 11 November 2010

C The Author(s) 2010. This article is published with open access at Springerlink.com

\begin{abstract}
Let $A$ be a central simple algebra with involution $\sigma$ of the first or second kind. Let $v$ be a valuation on the $\sigma$-fixed part $F$ of $Z(A)$. A $\sigma$-special $v$-gauge $g$ on $A$ is a kind of value function on $A$ extending $v$ on $F$, such that $g(\sigma(x) x)=2 g(x)$ for all $x$ in $A$. It is shown (under certain restrictions if the residue characteristic is 2) that if $v$ is Henselian, then there is a $\sigma$-special $v$-gauge $g$ if and only if $\sigma$ is anisotropic, and $g$ is unique. If $v$ is not Henselian, it is shown that there is a $\sigma$-special $v$-gauge $g$ if and only if $\sigma$ remains anisotropic after scalar extension from $F$ to the Henselization of $F$ with respect to $v$; when this occurs, $g$ is the unique $\sigma$-invariant $v$-gauge on $A$.
\end{abstract}

Mathematics Subject Classification (2000) $\quad$ 16W10 $16 \mathrm{~K} 20 \cdot 16 \mathrm{~W} 60 \cdot 11 \mathrm{E} 39$

\section{Introduction}

Valuations are a major tool for the study of the structure of division algebras. The purpose of this work is to introduce a notion that plays a similar role for central simple

J.-P. Tignol was partially supported by the F.R.S.-FNRS, Belgium. A.R. Wadsworth would like to thank J.-P. Tignol and UCL for their hospitality during several visits while this paper was developing.

\section{J.-P. Tignol}

Institute for Information and Communication Technologies, Electronics and Applied Mathematics, Université catholique de Louvain, 1348 Louvain-la-Neuve, Belgium

e-mail: jean-pierre.tignol@uclouvain.be

\section{A. R. Wadsworth $(\varangle)$}

Department of Mathematics, University of California, San Diego,

La Jolla, CA 92093-0112, USA

e-mail: arwadsworth@ucsd.edu 
algebras with involution, and to prove analogues for this notion to fundamental results on valuations on division algebras.

Since the definition of a (Schilling) valuation implies an absence of zero divisors, the only central simple algebras that can have valuations are division algebras. Given a division algebra $D$ finite-dimensional over its center $F$, it is natural to view valuations on $D$ as extensions of valuations on $F$, since valuations on fields are abundant and their theory is well-developed. But not every valuation $v$ on $F$ extends to $D$. In the extension question, Henselian valuations play a special role. Schilling proved in [17, pp. 53-54] that if $v$ on $F$ is Henselian, then $v$ has an extension to a valuation on $D$, and this extension is unique. Much later it was proved by Ershov [5] and Wadsworth [19] that for any valuation $v$ on $F, v$ extends to $D$ if and only if it satisfies a Henselian-like condition with respect to the field extensions of $F$ within $D$; they also proved that when $v$ extends to $D$ the extension is unique. Another fundamental criterion was proved by Morandi [14]: $v$ on $F$ extends to a valuation on $D$ if and only if $D$ remains a division algebra after scalar extension to the Henselization $F_{h}$ of $F$ for $v$. We will prove analogues for central simple algebras with involutions to these theorems of Schilling, Ershov-Wadsworth, and Morandi.

An involution on a central simple algebra $A$ is a ring-anti-automorphism $\sigma$ such that $\sigma^{2}=\mathrm{id}_{A}$. As Weil suggested in [20], the theory of central simple algebras with involution is a natural sibling to the theory of central simple algebras, since the associated automorphism groups are the basic types of classical groups. In each setting there is a notion of anisotropic object, corresponding to when the associated automorphism group is anisotropic as an algebraic group. The anisotropic central simple algebras are the division algebras. An involution $\sigma$ on a central simple algebra $A$ is anisotropic just when the equation $\sigma(x) x=0$ holds only for $x=0$. In earlier work [18] we have developed the theory of gauges, which are a kind of value functions for central simple algebras. (The definition of a gauge is recalled at the end of this introduction.) For a central simple algebra $A$ with involution $\sigma$, we define a $\sigma$-special gauge to be a gauge $\varphi$ on $A$ satisfying the condition ${ }^{1}$ that $\varphi(\sigma(x) x)=2 \varphi(x)$ for all $x \in A$. A $\sigma$-special gauge for an algebra with involution is our analogue to a valuation on a division algebra. If $A$ has a $\sigma$-special gauge, then $\sigma$ is easily seen to be anisotropic. If $v$ is a Henselian valuation on the $\sigma$-invariant part $F$ of $Z(A)$ and $\sigma$ is anisotropic, we show in Theorem 2.2 that there is a unique $\sigma$-invariant gauge $\varphi$ on $A$ extending $v$, and $\varphi$ is a $\sigma$-special gauge. When $v$ on $F$ is not Henselian, we show in Theorem 6.1 that there is a $\sigma$-special gauge $\varphi$ on $A$ extending $v$ if and only if the anisotropic involution $\sigma$ remains anisotropic after scalar extension to the Henselization of $F$ with respect to $v$; furthermore, there is only one such $\varphi$. Our results require tame ramification and exclude orthogonal involutions if the residue characteristic is 2; see the statements of Theorems 2.2 and 6.1 for the precise conditions required.

A gauge $\varphi$ on a central simple algebra $A$ induces a filtration on $A$ which yields an associated graded ring $\operatorname{gr}(A)$, analogous to what one has with a valuation on a field or a division ring. The graded structure is intrinsic to the definition of a gauge, and

\footnotetext{
${ }^{1}$ Notice the similarity with the definition of $C^{*}$-algebras, cf. [4, Déf. 1.3.1].
} 
is used heavily throughout this paper. The degree 0 part of $\operatorname{gr}(A)$, denoted $A_{0}$, is the residue ring of the "valuation ring" of $A$ determined by the gauge $\varphi ; A_{0}$ is always a semisimple $Z(A)_{0}$-algebra, but not simple in general. If $\sigma$ is an involution on $A$ and $\varphi$ is invariant under $\sigma$, then $\sigma$ induces involutions $\widetilde{\sigma}$ on $\operatorname{gr}(A)$ and $\sigma_{0}$ on $A_{0}$. We show in Proposition 1.1 and Remark 2.5(1) that a $\sigma$-invariant gauge $\varphi$ is $\sigma$-special if and only if $\widetilde{\sigma}$ is anisotropic, if and only if $\sigma_{0}$ is anisotropic. We also prove an analogue of a theorem of Springer: when the base field is Henselian, an involution $\sigma$ is isotropic if and only if its residue involution $\sigma_{0}$ is isotropic (Corollary 2.3). This criterion is applied to show that under specified valuation-theoretic conditions, an anisotropic involution remains anisotropic after certain scalar extensions(Corollary 3.6).

An outline of this paper is as follows: In Sect. 1, we discuss in general terms the compatibility of a value function with an involution, relating that notion to a compatibility condition between norms and hermitian forms defined in [16]. In Sect. 2, we restrict to the case of Henselian valuations and give the proofs of Theorem 2.2 and Corollary 2.3. Some applications to scalar extensions (in particular Corollary 3.6) are given in Sect. 3. Sections 4 and 5 prepare the ground for the extension of our results to the non-Henselian case in Sect. 6. The main problem is to analyze how the condition for the existence of a splitting base of a value function (which is a critical part of the definition of a gauge) behaves under restriction of scalars; this is done in Sect. 5. In Sect. 4, we investigate this condition for the composition of value functions. This is used in Sect. 6 in the proof of Theorem 6.1 by induction on the rank of valuations.

For the convenience of the reader, we now review the basic notions of value functions, norms, and gauges introduced in $[16,18]$. Throughout the paper, we fix a divisible totally ordered abelian group $\Gamma$, which will contain the values of all the valuations and the degrees of all the gradings we consider. Thus, a valued field $(F, v)$ is a pair consisting of a field $F$ and a valuation $v: F \rightarrow \Gamma \cup\{\infty\}$. The group $v\left(F^{\times}\right)$of values of $F$ is denoted by $\Gamma_{F}$, and the residue field by $\bar{F}$. We use analogous notation for valuations on division rings.

Let $(F, v)$ be a valued field. A $v$-value function on an $F$-vector space $V$ is a map $\alpha: V \rightarrow \Gamma \cup\{\infty\}$ such that

(i) $\alpha(x)=\infty$ if and only if $x=0$;

(ii) $\alpha(x+y) \geq \min (\alpha(x), \alpha(y))$ for $x, y \in V$;

(iii) $\alpha(x c)=\alpha(x)+v(c)$ for all $x \in V$ and $c \in F$.

The $v$-value function $\alpha$ is called a norm if $V$ is finite-dimensional and contains a base $\left(e_{i}\right)_{i=1}^{n}$ such that

$$
\alpha\left(\sum_{i=1}^{n} e_{i} c_{i}\right)=\min _{1 \leq i \leq n}\left(\alpha\left(e_{i} c_{i}\right)\right) \quad \text { for } c_{1}, \ldots, c_{n} \in F .
$$

Such a base is called a splitting base of $V$ for $\alpha$. A $v$-value function $\varphi$ on an $F$-algebra $A$ is surmultiplicative if $\varphi(1)=0$ and $\varphi(x y) \geq \varphi(x)+\varphi(y)$ for $x, y \in A$.

The valuation $v$ defines a filtration on $F$ : for $\gamma \in \Gamma$ we set

$$
\begin{aligned}
F_{\geq \gamma}= & \{x \in F \mid v(x) \geq \gamma\}, \quad F_{>\gamma}=\{x \in F \mid v(x)>\gamma\}, \\
& \text { and } \quad F_{\gamma}=F_{\geq \gamma} / F_{>\gamma} .
\end{aligned}
$$


The associated graded ring is

$$
\operatorname{gr}(F)=\bigoplus_{\gamma \in \Gamma} F_{\gamma}
$$

It is called a graded field because every nonzero homogeneous element in $\operatorname{gr}(F)$ is invertible. Likewise, every $v$-value function $\alpha$ on an $F$-vector space $V$ defines a filtration, and the associated graded structure $\operatorname{gr}_{\alpha}(V)$ is a graded module over $\operatorname{gr}(F)$, which we call a graded vector space. It is a free module, whose rank is called its dimension. The value function is a norm if and only if $\operatorname{dim}_{\operatorname{gr}(F)}\left(\operatorname{gr}_{\alpha}(V)\right)=\operatorname{dim}_{F}(V)<\infty$, see [16, Cor. 2.3]. Every nonzero element $x \in V$ has an image $\widetilde{x}$ in $\operatorname{gr}_{\alpha}(V)$ defined by

$$
\tilde{x}=x+V_{>\alpha(x)} \in V_{\alpha(x)} .
$$

We also set $\widetilde{0}=0 \in \operatorname{gr}_{\alpha}(V)$. If $\varphi$ is a surmultiplicative $v$-value function on an $F$-algebra $A$, then $\operatorname{gr}_{\varphi}(A)$ is an algebra over $\operatorname{gr}(F)$, in which multiplication is defined by

$$
\widetilde{a} \tilde{b}=a b+V_{>\varphi(a)+\varphi(b)}=\left\{\begin{array}{ll}
\widetilde{a b} & \text { if } \varphi(a b)=\varphi(a)+\varphi(b), \\
0 & \text { if } \varphi(a b)>\varphi(a)+\varphi(b),
\end{array} \text { for } a, b \in A\right.
$$

Now, suppose $A$ is a finite-dimensional simple $F$-algebra. We denote by $[A: F]$ its dimension and by $Z(A)$ its center. A surmultiplicative $v$-value function $\varphi$ on $A$ is called a $v$-gauge if it satisfies the following conditions:

(i) $\varphi$ is a $v$-norm, i.e., $[A: F]=\left[\operatorname{gr}_{\varphi}(A): \operatorname{gr}(F)\right]$;

(ii) $\operatorname{gr}_{\varphi}(A)$ is a graded semisimple $\operatorname{gr}(F)$-algebra, i.e., it does not contain any nonzero nilpotent homogeneous two-sided ideal.

The $v$-gauge $\varphi$ is said to be tame if $Z\left(\operatorname{gr}_{\varphi}(A)\right)=\operatorname{gr}_{\varphi}(Z(A))$ and $Z\left(\operatorname{gr}_{\varphi}(A)\right)$ is separable over $\operatorname{gr}(F)$. If the residue characteristic is 0 , then every $v$-gauge is tame, see [18, Cor. 3.6].

\section{Special gauges}

Let $(F, v)$ be a valued field and let $A$ be an $F$-algebra. An $F$-linear involution on $A$ is an $F$-linear map $\sigma: A \rightarrow A$ such that

(i) $\sigma(x+y)=\sigma(x)+\sigma(y)$ for $x, y \in A$;

(ii) $\sigma(x y)=\sigma(y) \sigma(x)$ for $x, y \in A$;

(iii) $\sigma^{2}(x)=x$ for $x \in A$.

(The $F$-linearity implies that $\left.\sigma\right|_{F}=\mathrm{id}_{F}$.) A surmultiplicative $v$-value function $\varphi: A \rightarrow \Gamma \cup\{\infty\}$ is said to be invariant under $\sigma$ if

$$
\varphi(\sigma(x))=\varphi(x) \quad \text { for all } x \in A \text {. }
$$


The involution then preserves the filtration on $A$ defined by $\varphi$. Therefore, it induces an involution $\widetilde{\sigma}$ on $\operatorname{gr}_{\varphi}(A)$ such that

$$
\widetilde{\sigma}(\widetilde{x})=\widetilde{\sigma(x)} \text { for all } x \in A \text {. }
$$

As in $[12, \S 6 . \mathrm{A}]$, we say that the involution $\sigma$ is anisotropic if there is no nonzero element $x \in A$ such that $\sigma(x) x=0$. Likewise, $\widetilde{\sigma}$ is said to be anisotropic if there is no nonzero homogeneous element $\xi \in \operatorname{gr}_{\varphi}(A)$ such that $\widetilde{\sigma}(\xi) \xi=0$. Clearly, if $\widetilde{\sigma}$ is anisotropic, then $\sigma$ is anisotropic.

Proposition 1.1 Let $\varphi$ be a surmultiplicative $v$-value function and $\sigma$ an F-linear involution on A. The following conditions are equivalent:

(a) $\varphi(\sigma(x) x)=2 \varphi(x)$ for all $x \in A$;

(b) $\varphi$ is invariant under $\sigma$, and $\widetilde{\sigma}$ is anisotropic.

They imply that if $x, y \in A$ satisfy $\sigma(x) y=0$ or $x \sigma(y)=0$, then

$$
\varphi(x+y)=\min (\varphi(x), \varphi(y)) .
$$

Moreover, when these equivalent conditions hold, $\sigma$ is anisotropic and the $\operatorname{gr}(F)$-algebra $\operatorname{gr}_{\varphi}(A)$ contains no nonzero homogeneous nil left or right ideal.

Proof (a) $\Rightarrow$ (b): If $\sigma(x) x=0$, then condition (a) implies that $\varphi(x)=\infty$, so $x=0$. Thus, $\sigma$ is anisotropic. By surmultiplicativity, we have

$$
\varphi(\sigma(x) x) \geq \varphi(\sigma(x))+\varphi(x) \text { for all } x \in A
$$

Therefore, (a) implies $\varphi(x) \geq \varphi(\sigma(x))$ for all $x \in A$. Substituting $\sigma(x)$ for $x$ in this inequality, we obtain $\varphi(\sigma(x)) \geq \varphi(x)$ for all $x \in A$. Therefore, $\varphi$ is invariant under $\sigma$, and condition (a) can be reformulated as $\varphi(\sigma(x) x)=\varphi(\sigma(x))+\varphi(x)$ for all $x \in A$. Thus, it implies

$$
\widetilde{\sigma(x)} \widetilde{x}=(\sigma(x) x)^{\sim} \quad \text { for all } x \in A
$$

whence $\widetilde{\sigma}$ is anisotropic, as $\sigma$ is anisotropic.

(b) $\Rightarrow$ (a): For all $x \in A$ we have

$$
\tilde{\sigma}(\tilde{x}) \tilde{x}= \begin{cases}(\sigma(x) x)^{\sim} & \text { if } \varphi(\sigma(x) x)=\varphi(\sigma(x))+\varphi(x), \\ 0 & \text { if } \varphi(\sigma(x) x)>\varphi(\sigma(x))+\varphi(x) .\end{cases}
$$

Condition (b) implies that the first case always occurs. Hence, for all $x$,

$$
\varphi(\sigma(x) x)=\varphi(\sigma(x))+\varphi(x)=2 \varphi(x)
$$


For the rest of the proof, assume (a) and (b) hold. Then clearly $\sigma$ is aniso-tropic. Also, for $x, y \in A$ we have by surmultiplicativity

$$
\varphi(\sigma(x) \cdot(x+y)) \geq \varphi(\sigma(x))+\varphi(x+y)=\varphi(x)+\varphi(x+y) .
$$

If $\sigma(x) y=0$, then

$$
\varphi(\sigma(x) \cdot(x+y))=\varphi(\sigma(x) x)=2 \varphi(x)
$$

By combining (1.3) and (1.4), we obtain $\varphi(x) \geq \varphi(x+y)$. Similarly, by interchanging $x$ and $y$ we get $\varphi(y) \geq \varphi(x+y)$, hence

$$
\min (\varphi(x), \varphi(y)) \geq \varphi(x+y) .
$$

The reverse inequality holds by definition of a value function, hence (1.2) is proved when $\sigma(x) y=0$. If $x \sigma(y)=0$, we substitute $\sigma(x)$ for $x$ and $\sigma(y)$ for $y$ in the arguments above, obtaining

$$
\varphi(\sigma(x)+\sigma(y))=\min (\varphi(\sigma(x)), \varphi(\sigma(y))) .
$$

Equation (1.2) follows since $\varphi \circ \sigma=\varphi$.

To complete the proof, suppose $\mathrm{I} \subset \operatorname{gr}_{\varphi}(A)$ is a homogeneous nil left (resp. right) ideal and $\xi \in \mathrm{I}$ is a nonzero homogeneous element. Let $\eta=\widetilde{\sigma}(\xi) \xi($ resp. $\eta=\xi \widetilde{\sigma}(\xi))$. Then $\eta \in \mathrm{I}$ is $\widetilde{\sigma}$-symmetric, homogeneous, and nonzero since $\widetilde{\sigma}$ is anisotropic. Since $\mathrm{I}$ is nil, we may find $k \geq 1$ such that $\eta^{k} \neq 0$ and $\eta^{k+1}=0$. For $\zeta=\eta^{k}$ we have

$$
\widetilde{\sigma}(\zeta) \zeta=\zeta^{2}=\eta^{2 k}=0
$$

so $\zeta=0$, a contradiction.

Definition 1.2 A surmultiplicative $v$-value function $\varphi$ on a central simple algebra $A$ with involution $\sigma$ is called $\sigma$-special if it satisfies the conditions (a) and (b) of Proposition 1.1.

For use in Sects. 3 and 6, we record how involution invariance of value functions behaves with respect to tensor products. Recall from [18, Prop. 1.23, (1.25)] that if $V$ is a finite-dimensional $F$-vector space with a $v$-norm $\alpha$ and $W$ is an $F$-vector space with $v$-value function $\beta$, then there is a $v$-value function $\alpha \otimes \beta$ on $V \otimes_{F} W$ uniquely determined by the condition that the map $(x \otimes y)^{\sim} \mapsto \widetilde{x} \otimes \widetilde{y}$ (for $x \in V$ and $y \in W$ ) defines an isomorphism of graded vector spaces

$$
\Omega: \operatorname{gr}_{\alpha \otimes \beta}\left(V \otimes_{F} W\right) \stackrel{\sim}{\rightarrow} \operatorname{gr}_{\alpha}(V) \otimes_{\operatorname{gr}(F)} \operatorname{gr}_{\beta}(W)
$$

In particular,

$$
(\alpha \otimes \beta)(x \otimes y)=\alpha(x)+\beta(y) \text { for all } x \in V \text { and } y \in W \text {. }
$$


The value function $\alpha \otimes \beta$ can be defined as follows: take any splitting base $\left(e_{i}\right)_{i=1}^{n}$ for $\alpha$ on $V$; then,

$$
(\alpha \otimes \beta)\left(\sum_{i=1}^{n} e_{i} \otimes y_{i}\right)=\min _{1 \leq i \leq n}\left(\alpha\left(e_{i}\right)+\beta\left(y_{i}\right)\right) \quad \text { for any } y_{1}, \ldots, y_{n} \in W
$$

Furthermore, analogous to [18, Cor. 1.26], if $(W, \beta)$ is a valued field extending $(F, v)$,

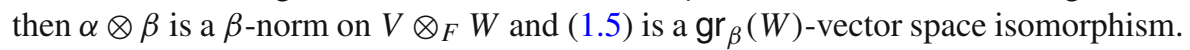

Proposition 1.3 Let $\sigma$ and $\tau$ be $F$-linear involutions on $F$-algebras $A$ and $B$ respectively, and let $\varphi$ (resp. $\psi$ ) be a surmultiplicative $v$-value function on $A$ (resp. B) invariant under $\sigma$ (resp. $\tau$ ). Suppose $A$ is finite-dimensional and $\varphi$ is a v-norm. Then, $\varphi \otimes \psi$ is a surmultiplicative $v$-value function on $A \otimes_{F} B$ invariant under the involution $\sigma \otimes \tau$, and the canonical isomorphism $\Omega$ of (1.5) is an isomorphism of graded $\operatorname{gr}(F)$-algebras with involution,

$$
\left(\operatorname{gr}_{\varphi \otimes \psi}\left(A \otimes_{F} B\right), \widetilde{\sigma \otimes \tau}\right) \stackrel{\sim}{\rightarrow}\left(\operatorname{gr}_{\varphi}(A) \otimes \operatorname{gr}(F) \operatorname{gr}_{\psi}(B), \widetilde{\sigma} \otimes \widetilde{\tau}\right)
$$

Proof Let $\left(e_{i}\right)_{i=1}^{n}$ be a splitting base of $A$ for $\varphi$. For $x, y \in A \otimes_{F} B$ we may write

$$
x=\sum_{i=1}^{n} e_{i} \otimes x_{i} \quad \text { and } \quad y=\sum_{j=1}^{n} e_{j} \otimes y_{j} \quad \text { for some } x_{1}, \ldots, y_{n} \in B .
$$

Then,

$$
\begin{aligned}
(\varphi \otimes \psi)(x y) & =(\varphi \otimes \psi)\left(\sum_{i, j} e_{i} e_{j} \otimes x_{i} x_{j}\right) \geq \min _{1 \leq i, j \leq n}\left((\varphi \otimes \psi)\left(e_{i} e_{j} \otimes x_{i} y_{j}\right)\right) \\
& =\min _{i, j}\left(\varphi\left(e_{i} e_{j}\right)+\psi\left(x_{i} y_{j}\right)\right) .
\end{aligned}
$$

Since $\varphi$ and $\psi$ are surmultiplicative, we have $\varphi\left(e_{i} e_{j}\right) \geq \varphi\left(e_{i}\right)+\varphi\left(e_{j}\right)$ and $\psi\left(x_{i} y_{j}\right) \geq$ $\psi\left(x_{i}\right)+\psi\left(y_{j}\right)$, hence

$$
\begin{aligned}
(\varphi \otimes \psi)(x y) & \geq \min _{i, j}\left(\varphi\left(e_{i}\right)+\varphi\left(e_{j}\right)+\psi\left(x_{i}\right)+\psi\left(y_{j}\right)\right) \\
& =\min _{i}\left(\varphi\left(e_{i}\right)+\psi\left(x_{i}\right)\right)+\min _{j}\left(\varphi\left(e_{j}\right)+\psi\left(y_{j}\right)\right) .
\end{aligned}
$$

The last line is $(\varphi \otimes \psi)(x)+(\varphi \otimes \psi)(y)$, so

$$
(\varphi \otimes \psi)(x y) \geq(\varphi \otimes \psi)(x)+(\varphi \otimes \psi)(y)
$$

Since moreover $(\varphi \otimes \psi)(1 \otimes 1)=\varphi(1)+\psi(1)=0$, surmultiplicativity of $\varphi \otimes \psi$ is proved.

To show that the $g r_{F}$-vector space isomorphism $\Omega$ is a ring isomorphism, we check this for $\Omega^{-1}$. The $F$-algebra homomorphisms $\iota_{A}: A \rightarrow A \otimes_{F} B, a \mapsto a \otimes 1$ and 
$\iota_{B}: B \rightarrow A \otimes_{F} B, b \mapsto 1 \otimes b$, are value-preserving. Hence, they induce $\operatorname{gr}(F)$ algebra homomorphisms $\tau_{A}: \operatorname{gr}_{\alpha}(A) \rightarrow \operatorname{gr}_{\alpha \otimes \beta}\left(A \otimes_{F} B\right)$ given by $\widetilde{a} \mapsto \widehat{a \otimes 1}$, and $\tau_{B}: \operatorname{gr}_{\beta}(B) \rightarrow \operatorname{gr}_{\alpha \otimes \beta}\left(A \otimes_{F} B\right)$ given by $\widetilde{b} \mapsto \widetilde{1 \otimes b}$. For any $a \in A$ and $b \in B$, we have from (1.6),

$$
\begin{aligned}
\widetilde{a \otimes 1} \cdot \widetilde{1 \otimes b} & =[(a \otimes 1) \cdot(1 \otimes b)]^{\sim}=\widetilde{a \otimes b}=[(1 \otimes b) \cdot(a \otimes 1)]^{\sim} \\
& =\widetilde{1 \otimes b} \cdot \widetilde{a \otimes 1} .
\end{aligned}
$$

Thus, $\operatorname{im}\left(\tau_{B}\right)$ centralizes $\operatorname{im}\left(\tau_{A}\right)$ in $\operatorname{gr}_{\alpha \otimes \beta}\left(A \otimes_{F} B\right)$. So, there is an induced $\operatorname{gr}(F)$ -

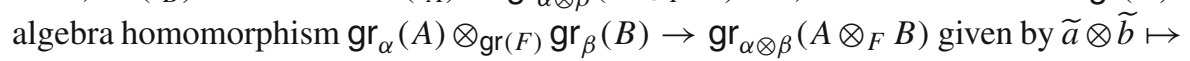
$\widetilde{a \otimes 1} \cdot \widetilde{1 \otimes b}=\widetilde{a \otimes b}$. The description of $\Omega$ preceding (1.5) shows that this algebra homomorphism is $\Omega^{-1}$.

To prove $\varphi \otimes \psi$ is invariant under $\sigma \otimes \tau$, we first show that $\left(\sigma\left(e_{i}\right)\right)_{i=1}^{n}$ also is a splitting base of $A$ for $\varphi$. Take any $c_{1}, \ldots, c_{n} \in F$. Then, as $\varphi$ is invariant under $\sigma$ and the $c_{i}$ are central in $A$ and fixed under $\sigma$,

$$
\begin{aligned}
\varphi\left(\sum_{i=1}^{n} \sigma\left(e_{i}\right) c_{i}\right) & =\varphi\left(\sigma\left(\sum_{i=1}^{n} \sigma\left(e_{i}\right) c_{i}\right)\right)=\varphi\left(\sum_{i=1}^{n} e_{i} c_{i}\right) \\
& =\min _{1 \leq i \leq n}\left(\varphi\left(e_{i}\right)+v\left(c_{i}\right)\right)=\min _{1 \leq i \leq n}\left(\varphi\left(\sigma\left(e_{i}\right)\right)+v\left(c_{i}\right)\right)
\end{aligned}
$$

Thus, $\left(\sigma\left(e_{i}\right)\right)_{i=1}^{n}$ is a splitting base for $\varphi$. With the notation above, we then have

$$
\begin{aligned}
(\varphi \otimes \psi)((\sigma \otimes \tau)(x)) & =(\varphi \otimes \psi)\left(\sum_{i=1}^{n} \sigma\left(e_{i}\right) \otimes \tau\left(x_{i}\right)\right) \\
& =\min _{1 \leq i \leq n}\left(\varphi\left(\sigma\left(e_{i}\right)\right)+\psi\left(\tau\left(x_{i}\right)\right)\right)
\end{aligned}
$$

Since $\varphi$ is invariant under $\sigma$ and $\psi$ under $\tau$, we have

$$
\min _{1 \leq i \leq n}\left(\varphi\left(\sigma\left(e_{i}\right)\right)+\psi\left(\tau\left(x_{i}\right)\right)\right)=\min _{1 \leq i \leq n}\left(\varphi\left(e_{i}\right)+\psi\left(x_{i}\right)\right)=(\varphi \otimes \psi)(x)
$$

Therefore, $\varphi \otimes \psi$ is invariant under $\sigma \otimes \tau$. To complete the proof, observe that for $a \in A$ and $b \in B$ we have

$$
\widetilde{\sigma \otimes \tau}(\widetilde{a \otimes b})=(\sigma(a) \otimes \tau(b))^{\sim}=(\widetilde{\sigma} \otimes \tilde{\tau})(\widetilde{a} \otimes \widetilde{b}),
$$

hence the involution $\tilde{\sigma} \otimes \tilde{\tau}$ corresponds to $\widetilde{\sigma \otimes \tau}$ under the canonical isomorphism (1.5).

The following special case will be particularly useful:

Corollary 1.4 Let $A$ be a finite-dimensional $F$-algebra with an $F$-linear involution $\sigma$ and let $\left(K, v_{K}\right)$ be any valued field extension of $(F, v)$. If $\varphi$ is a surmultiplicative $v$-norm on A which is invariant under $\sigma$, then $\varphi \otimes v_{K}$ is a surmultiplicative $v_{K}$-norm 
on $A \otimes_{F} K$ invariant under the involution $\sigma \otimes \operatorname{id}_{K}$, and $\left(\sigma \otimes \operatorname{id}_{K}\right)^{\sim} \cong \widetilde{\sigma} \otimes \operatorname{id}_{\operatorname{gr}(K)}$ under the canonical isomorphism (1.5).

Proof It suffices to note that $\widetilde{\mathrm{id}_{K}}=\operatorname{id}_{\operatorname{gr}(K)}$ and that the canonical isomorphism is an isomorphism of $\operatorname{gr}(K)$-algebras, see [18, Cor. 1.26].

Now, assume $A$ is simple and finite-dimensional, and let $n=\operatorname{deg} A$, so $[A: Z(A)]=n^{2}$. Recall from [12] that involutions on $A$ are classified into two kinds and three types: an involution $\sigma$ is of the first kind if $\left.\sigma\right|_{Z(A)}=\mathrm{id}_{Z(A)}$; otherwise it is of the second kind. Involutions of the second kind are also said to be of unitary type (or simply unitary). To define the type of an involution $\sigma$ of the first kind we consider the subspaces of symmetric and of symmetrized elements in $A$, defined by

$$
\operatorname{Sym}(A, \sigma)=\{x \in A \mid \sigma(x)=x\} \text { and } \operatorname{Symd}(A, \sigma)=\{x+\sigma(x) \mid x \in A\} .
$$

The involution $\sigma$ is of symplectic type (or simply symplectic) if either char $(F) \neq 2$ and $\operatorname{dim}_{Z(A)} \operatorname{Sym}(A, \sigma)=\frac{1}{2} n(n-1)$ or $\operatorname{char}(F)=2$ and $1 \in \operatorname{Symd}(A, \sigma)$. Involutions of the first kind that are not symplectic are said to be of orthogonal type (or simply orthogonal). If $\sigma$ is orthogonal, then $\operatorname{dim}_{Z(A)} \operatorname{Sym}(A, \sigma)=\frac{1}{2} n(n+1)$. The same terminology is used for involutions on graded simple algebras.

Proposition 1.5 Let $\sigma$ be an F-linear involution on a finite-dimensional simple $F$-algebra $A$ and let $g$ be a tame $v$-gauge on $A$ that is invariant under $\sigma$. Suppose $F$ is the subfield of $Z(A)$ fixed under $\sigma$.

If $\sigma$ is unitary, two cases may arise:

- if the valuation $v$ extends uniquely from $F$ to $Z(A)$, then $\operatorname{gr}_{g}(A)$ is a graded simple $\operatorname{gr}(F)$-algebra and $\widetilde{\sigma}$ is a unitary involution;

- if the valuation $v$ has two different extensions to $Z(A)$, then $\operatorname{gr}_{g}(A)$ is a direct product of two graded central simple $\operatorname{gr}(F)$-algebras, which are exchanged under $\widetilde{\sigma}$.

If $\sigma$ is symplectic, then $\widetilde{\sigma}$ is a symplectic involution on the graded central simple $\operatorname{gr}(F)$-algebra $\operatorname{gr}_{g}(A)$.

If $\sigma$ is orthogonal and $\operatorname{char}(\bar{F}) \neq 2$, then $\widetilde{\sigma}$ is an orthogonal involution on the graded central simple $\mathrm{gr}(F)$-algebra $\operatorname{gr}_{g}(A)$.

Proof Suppose first that $\sigma$ is unitary, so $Z(A) / F$ is a quadratic extension. By [18, Cor. 2.5], the number of simple components of $\operatorname{gr}(A)$ equals the number of extensions of $v$ to $Z(A)$. Therefore, to complete the description of $\widetilde{\sigma}$ it suffices to show that $\widetilde{\sigma}$ does not identically fix $Z(\operatorname{gr}(A))=\operatorname{gr}(Z(A))$. Since the Galois group $\mathcal{G}(Z(A) / F)$ acts transitively on the set of extensions of $v$ to $Z(A)$, see [9, Th. 3.2.15, p. 64], if there are two such extensions, then $\left.\sigma\right|_{Z(A)}$ must permute them; then $\widetilde{\sigma}$ permutes the corresponding components of $\operatorname{gr}(Z(A))$. So, we may assume that $v$ has a unique extension to $Z(A)$. Then, $\operatorname{gr}(Z(A))$ is a graded field separable over $\operatorname{gr}(F)$, and $[\operatorname{gr}(Z(A)): \operatorname{gr}(F)]=[Z(A): F]=2$ since $\left.g\right|_{Z(A)}$ is a norm, by [16, Prop. 2.5]. If $\operatorname{char}(\bar{F}) \neq 2$ we can find $z \in Z(A)$ nonzero such that $\sigma(z)=-z$, hence $\widetilde{\sigma}(\widetilde{z})=-\widetilde{z} \neq \widetilde{z}$. If $\operatorname{char}(\bar{F})=2$ the separability of $\operatorname{gr}(Z(A))$ over $\operatorname{gr}(F)$ implies by 
[10, Th. 3.11, Def. 3.4] that $\Gamma_{Z(A)}=\Gamma_{F}$ and $Z(A)_{0}$ is separable over $F_{0}$; furthermore, $\left[Z(A)_{0}: F_{0}\right]=[Z(A): F]=2$ since $\left.g\right|_{Z(A)}$ is a norm, by [16, Prop. 2.5]. So $Z(A)$ is unramified Galois over $F$, hence the non-trivial automorphism $\left.\sigma\right|_{Z(A)}$ induces a nontrivial automorphism of the residue algebra $Z(A)_{0}$, by [8, Th. 19.6, p. 124], showing that $\widetilde{\sigma} \operatorname{gr}(Z(A))$ is nontrivial.

Suppose next that $\sigma$ is of the first kind, so $Z(A)=F$. For $x \in A$ we have $\tilde{x}+\tilde{\sigma}(\tilde{x})=(x+\sigma(x))^{\sim}$ or 0 . On the other hand, $\sigma(x)=x$ implies $\widetilde{\sigma}(\tilde{x})=\tilde{x}$. Therefore, the following inclusions are clear:

$$
\operatorname{gr}(\operatorname{Sym}(A, \sigma)) \subseteq \operatorname{Sym}(\operatorname{gr}(A), \widetilde{\sigma}), \quad \operatorname{Symd}(\operatorname{gr}(A), \widetilde{\sigma}) \subseteq \operatorname{gr}(\operatorname{Symd}(A, \sigma))
$$

If $\operatorname{char}(\bar{F}) \neq 2$ (hence $\operatorname{char}(F) \neq 2$ ) we have

$$
\operatorname{Sym}(A, \sigma)=\operatorname{Symd}(A, \sigma) \text { and } \operatorname{Sym}(\operatorname{gr}(A), \widetilde{\sigma})=\operatorname{Symd}(\operatorname{gr}(A), \widetilde{\sigma}),
$$

so the inclusions in (1.7) above yield $\operatorname{gr}(\operatorname{Sym}(A, \sigma))=\operatorname{Sym}(\operatorname{gr}(A), \widetilde{\sigma})$. Since the type of an involution can be determined from the dimension of the space of symmetric elements, it follows that $\widetilde{\sigma}$ has the same type as $\sigma$.

To complete the proof, suppose $\operatorname{char}(\bar{F})=2$ and $\sigma$ is symplectic, and let $n=\operatorname{deg} A$. Since $\widetilde{\sigma}$ is of the first kind we have

$$
\operatorname{dim}_{\operatorname{gr}(F)} \operatorname{Symd}(\operatorname{gr}(A), \widetilde{\sigma})=\frac{1}{2} n(n-1) .
$$

On the other hand, since $\sigma$ is symplectic we have

$$
\operatorname{dim}_{F} \operatorname{Symd}(A, \sigma)=\frac{1}{2} n(n-1)
$$

(independently of whether $\operatorname{char}(F)=2$ ). Since $g$ is a norm we have

$$
\operatorname{dim}_{F} \operatorname{Symd}(A, \sigma)=\operatorname{dim}_{\operatorname{gr}(F)} \operatorname{gr}(\operatorname{Symd}(A, \sigma)),
$$

hence $\operatorname{Symd}(\operatorname{gr}(A), \widetilde{\sigma})=\operatorname{gr}(\operatorname{Symd}(A, \sigma))$. Since $1 \in \operatorname{Symd}(A, \sigma)$, it follows that $\widetilde{1} \in \operatorname{Symd}(\operatorname{gr}(A), \widetilde{\sigma})$, hence $\widetilde{\sigma}$ is symplectic.

Remark 1.6 If $\sigma$ is orthogonal and $\operatorname{char}(\bar{F})=2$, the involution $\widetilde{\sigma}$ may be symplectic, as the following example shows: let $(F, v)$ be a valued field with $\operatorname{char}(F)=0$ and $\operatorname{char}(\bar{F})=2$, and let $A=M_{2}(F)$. Define an orthogonal involution $\sigma$ on $A$ by

$$
\sigma\left(\begin{array}{ll}
a & b \\
c & d
\end{array}\right)=\left(\begin{array}{ll}
d & b \\
c & a
\end{array}\right)
$$

and a $v$-gauge $g$ by

$$
g\left(\begin{array}{ll}
a & b \\
c & d
\end{array}\right)=\min (v(a), v(b), v(c), v(d))
$$


This gauge is clearly invariant under $\sigma$. We have $\operatorname{gr}_{g}(A)=M_{2}(\operatorname{gr}(F))$ with the entrywise grading, and

$$
\left.\left(\begin{array}{ll}
1 & 0 \\
0 & 1
\end{array}\right)=\left(\begin{array}{ll}
1 & 0 \\
0 & 0
\end{array}\right)+\widetilde{\sigma}\left(\begin{array}{ll}
1 & 0 \\
0 & 0
\end{array}\right) \in \operatorname{Symd}_{g}(A), \widetilde{\sigma}\right)
$$

Therefore, $\widetilde{\sigma}$ is symplectic.

Henceforth, we systematically avoid orthogonal involutions in characteristic 2.

In [16, Sect. 3], a notion of compatibility is defined between norms and hermitian forms. In the rest of this section, we relate that notion of compatibility with the invariance of value functions under involutions.

Let $D$ be a finite-dimensional division $F$-algebra with an $F$-linear involution $\tau$. Suppose $v$ extends to a valuation $w$ on $D$ invariant under $\tau$ and let $V$ be a finite-dimensional right $D$-vector space. Consider a nondegenerate hermitian form $h: V \times V \rightarrow D$ with respect to $\tau$, and a $w$-norm $\alpha$ on $V$. The dual norm $\alpha^{\sharp}$ is defined by

$$
\alpha^{\sharp}(x)=\min \{w(h(x, y))-\alpha(y) \mid y \in V, y \neq 0\} \quad \text { for } x \in V,
$$

see [16, Sect. 3]. The norm $\alpha$ is said to be compatible with $h$ if and only if $\alpha^{\sharp}=\alpha$ (see [16, Prop. 3.5]). This is the condition needed in order for $h$ to induce a nondegenerate graded hermitian form on $\operatorname{gr}_{\alpha}(V)$. On the simple algebra $\operatorname{End}_{D}(V)$ there is the involution $\operatorname{ad}_{h}$ adjoint to $h$, defined by

$$
h\left(\operatorname{ad}_{h}(f)(x), y\right)=h(x, f(y)) \quad \text { for all } x, y \in V .
$$

There is also the well-defined surmultiplicative $v$-value function $\operatorname{End}(\alpha)$ on $\operatorname{End}_{D}(V)$ defined by

$$
\operatorname{End}(\alpha)(f)=\min \{\alpha(f(x))-\alpha(x) \mid x \in V, x \neq 0\}
$$

Recall that $\operatorname{End}(\alpha)$ is a $v$-gauge if and only if $w$ on $D$ is defectless over $v$, see [18, Prop. 1.19].

Proposition 1.7 The value functions $\operatorname{End}(\alpha)$ and $\operatorname{End}\left(\alpha^{\sharp}\right)$ are related by

$$
\operatorname{End}(\alpha) \circ \operatorname{ad}_{h}=\operatorname{End}\left(\alpha^{\sharp}\right) .
$$

Moreover, the following conditions are equivalent:

(a) $\operatorname{End}(\alpha)$ is invariant under $\operatorname{ad}_{h}$;

(b) $\operatorname{End}\left(\alpha^{\sharp}\right)=\operatorname{End}(\alpha)$;

(c) $\alpha-\alpha^{\sharp}$ is constant on $V$;

(d) there is a constant $\gamma \in \Gamma$ such that $\alpha-\gamma$ is compatible with $h$. 
Proof Let $\left(e_{i}\right)_{i=1}^{n}$ be a splitting base of $V$ for $\alpha$. The $h$-dual base $\left(e_{i}^{\sharp}\right)_{i=1}^{n}$ for $V$ is a splitting base for $\alpha^{\sharp}$, by [16, Lemma 3.4]. Fix some $f \in \operatorname{End}_{D} V$, and let

$$
f\left(e_{j}^{\sharp}\right)=\sum_{i=1}^{n} e_{i}^{\sharp} d_{i j} \quad \text { for some } d_{i j} \in D .
$$

Then, computation yields

$$
\operatorname{ad}_{h}(f)\left(e_{j}\right)=\sum_{i=1}^{n} e_{i} \tau\left(d_{j i}\right)
$$

We may compute $\operatorname{End}\left(\alpha^{\sharp}\right)(f)$ using the splitting base $\left(e_{i}^{\sharp}\right)_{i=1}^{n}$, and $\operatorname{End}(\alpha)\left(\operatorname{ad}_{h}(f)\right)$ using the splitting base $\left(e_{i}\right)_{i=1}^{n}$, obtaining

$$
\begin{aligned}
\operatorname{End}\left(\alpha^{\sharp}\right)(f) & =\min _{1 \leq i, j \leq n}\left(\alpha^{\sharp}\left(e_{i}^{\sharp}\right)+w\left(d_{i j}\right)-\alpha^{\sharp}\left(e_{j}^{\sharp}\right)\right), \\
\operatorname{End}(\alpha)\left(\operatorname{ad}_{h}(f)\right) & =\min _{1 \leq i, j \leq n}\left(\alpha\left(e_{i}\right)+w\left(\tau\left(d_{j i}\right)\right)-\alpha\left(e_{j}\right)\right) .
\end{aligned}
$$

Equation (1.9) follows since $\alpha^{\sharp}\left(e_{i}^{\sharp}\right)=-\alpha\left(e_{i}\right)$, see [16, Lemma 3.4].

The equivalence of (a) and (b) readily follows from (1.9), and the equivalence of (b) and (c) from [18, Prop. 1.22].

(c) $\Leftrightarrow$ (d): By the definition of the dual norm in (1.8), for any constant $\gamma$ in the divisible group $\Gamma,(\alpha-\gamma)^{\sharp}=\alpha^{\sharp}+\gamma$. Therefore, $\alpha-\gamma$ is compatible with $h$ if and only if $(\alpha-\gamma)^{\sharp}=\alpha-\gamma$, which holds if and only if $\alpha-\alpha^{\sharp}=2 \gamma$.

Suppose the equivalent conditions of Proposition 1.7 hold, and write simply $g_{\alpha}$ for $\operatorname{End}(\alpha)$. Recall from [18, Prop. 1.19] that the graded algebra $\operatorname{gr}_{g_{\alpha}}\left(\operatorname{End}_{D} V\right)$ may be identified with $\operatorname{End}_{\operatorname{gr}(D)}\left(\operatorname{gr}_{\alpha}(V)\right)$ so that for $f \in \operatorname{End}_{D} V$ the element $\widetilde{f} \in \operatorname{gr}_{g_{\alpha}}\left(\operatorname{End}_{D} V\right)$ is viewed as the map $\widetilde{f}: \operatorname{gr}_{\alpha}(V) \rightarrow \operatorname{gr}_{\alpha}(V)$ defined by

$$
\widetilde{f}(\tilde{x})= \begin{cases}\widetilde{f(x)} & \text { if } \alpha(f(x))=\alpha(x)+g_{\alpha}(f), \\ 0 & \text { if } \alpha(f(x))>\alpha(x)+g_{\alpha}(f)\end{cases}
$$

On the other hand, after adding a constant if necessary, we may assume $\alpha$ is compatible with $h$; hence we may define a graded hermitian form

$$
\widetilde{h}: \operatorname{gr}_{\alpha}(V) \times \operatorname{gr}_{\alpha}(V) \rightarrow \operatorname{gr}_{w}(D)
$$

(with respect to the involution $\widetilde{\tau}$ ) as follows: for $x, y \in V$,

$$
\widetilde{h}(\tilde{x}, \tilde{y})=h(x, y)+D_{>\alpha(x)+\alpha(y)}= \begin{cases}\widehat{h(x, y)} & \text { if } w(h(x, y))=\alpha(x)+\alpha(y), \\ 0 & \text { if } w(h(x, y))>\alpha(x)+\alpha(y) .\end{cases}
$$

This hermitian form is well-defined and nondegenerate (cf. [16], Remark 3.2), and we may therefore consider the adjoint involution $\operatorname{ad}_{\widetilde{h}}$ on $\operatorname{End}_{\operatorname{gr}_{w}(D)}\left(\operatorname{gr}_{\alpha}(V)\right)=$ $\operatorname{gr}_{g_{\alpha}}\left(\operatorname{End}_{D} V\right)$. 
Proposition 1.8 Assuming $\alpha$ is compatible with h, the involution $\widetilde{a d}_{h}$ on $\operatorname{gr}_{g_{\alpha}}\left(\operatorname{End}_{D} V\right)$ is the adjoint involution of $\widetilde{h}$ under the identification above; i.e.,

$$
\widetilde{\operatorname{ad}}_{h}=\operatorname{ad}_{\tilde{h}} .
$$

In particular, the value function $g_{\alpha}=\operatorname{End}(\alpha)$ is $\sigma$-special (see Definition 1.2) if and only if $\widetilde{h}$ is anisotropic.

Proof To verify the equality of graded involutions, it suffices to show, for all $x, y \in V$ and $f \in \operatorname{End}_{D} V$,

$$
\widetilde{h}\left(\operatorname{ad}_{\tilde{h}}(\widetilde{f})(\widetilde{x}), \widetilde{y}\right)=\widetilde{h}\left(\widetilde{\operatorname{ad}}_{h}(\widetilde{f})(\widetilde{x}), \widetilde{y}\right)
$$

From the definition of $\operatorname{ad}_{\tilde{h}}$, it is equivalent to prove

$$
\widetilde{h}(\tilde{x}, \tilde{f}(\widetilde{y}))=\widetilde{h}\left(\tilde{\operatorname{ad}}_{h}(\tilde{f})(\widetilde{x}), \tilde{y}\right) .
$$

Since $\alpha$ is compatible with $h$, Proposition 1.7 shows $g_{\alpha}$ is invariant under $\operatorname{ad}_{h}$; hence, $g_{\alpha}\left(\operatorname{ad}_{h}(f)\right)=g_{\alpha}(f)$. Therefore, each side of (1.10) lies in $D_{\epsilon}$, where $\epsilon=\alpha(x)+\alpha(y)$ $+g_{\alpha}(f)$. Suppose $w(h(x, f(y)))=\epsilon$. Then, necessarily $\alpha(f(y))=g_{\alpha}(f)+$ $\alpha(y)$, and the left side of (1.10) equals $h(x, f(y))^{\sim}$. But since $h(x, f(y))=$ $h\left(\operatorname{ad}_{h}(f)(x), y\right)$, we then also have

$$
g_{\alpha}\left(\operatorname{ad}_{h}(f)(x)\right)=g_{\alpha}\left(\operatorname{ad}_{h}(f)\right)+\alpha(x)
$$

and the right side of (1.10) becomes $h\left(\operatorname{ad}_{h}(f)(x), y\right)^{\sim}$. So, (1.10) then holds. But, if $w(h(x, f(y)))>\epsilon$, then each side of (1.10) is 0 . Thus, the equality (1.10) holds in all cases, so that $\widetilde{a d}_{h}=\operatorname{ad}_{\tilde{h}}$.

Since $g_{\alpha}$ is invariant under $\mathrm{ad}_{h}$, Proposition 1.1(b) holds if and only if $\widetilde{\mathrm{ad}}_{h}$ is anisotropic. But, the involution $\widetilde{\operatorname{ad}}_{h}=\operatorname{ad}_{\tilde{h}}$ is anisotropic if and only if its associated graded hermitian form $\widetilde{h}$ is anisotropic. This is proved analogously to the ungraded case [12, Sect. 6.A], using the fact that $\widetilde{h}$ is anisotropic if and only if $\widetilde{h}(\widetilde{x}, \widetilde{x}) \neq \widetilde{0}$ for all nonzero $x \in V$, as remarked in [16, p. 101].

\section{Henselian valuations}

Throughout this section, $(F, v)$ is a Henselian valued field and $A$ is a finitedimensional simple $F$-algebra with an involution $\sigma$. We let $K=Z(A)$ and assume $F$ is the subfield of $K$ fixed by $\sigma$. (Thus, $A$ is central over $F$ if $\left.\sigma\right|_{Z(A)}=\operatorname{id}_{Z(A)}$ ). We assume $A$ is tame over $F$, which means that $A$ is split by the maximal tamely ramified extension of $K$, and that $K$ is tame over $F$. Moreover, if $\operatorname{char}(\bar{F})=2$ we assume $\sigma$ is not an orthogonal involution.

Proposition 2.1 With the hypotheses above, every v-gauge on A is tame. Furthermore, there exist $v$-gauges on A that are invariant under $\sigma$. 
Proof We may represent $A=\operatorname{End}_{D} V$ for some finite-dimensional right vector space $V$ over a central division $K$-algebra $D$. Since $v$ is Henselian, it extends uniquely to a valuation $w$ on $D$ (see for instance [17, p. 53, Th. 9] or [19, Th.]). Since $A$ is tame over $F$, by [18, Prop. 1.19] $D$ must also be tame over $F$; hence by [18, Prop. 1.12 and 1.13] $w$ is a tame $v$-gauge. Therefore, every $v$-gauge on $A$ is tame, by [18, Th. 3.1].

If $A$ is split and $\sigma$ is symplectic, then $\sigma=\operatorname{ad}_{b}$ for some alternating bilinear form $b$ on $V$, see $\left[12\right.$, Sect. 4.A]. Choose a symplectic base $\mathcal{B}=\left(e_{i}, f_{i}\right)_{i=1}^{n}$ of $V$ for $b$ and define a $v$-norm $\alpha$ on $V$ by

$$
\alpha\left(\sum_{i=1}^{n} e_{i} \lambda_{i}+f_{i} \mu_{i}\right)=\min _{1 \leq i \leq n}\left(v\left(\lambda_{i}\right), v\left(\mu_{i}\right)\right) \quad \text { for } \lambda_{1}, \ldots, \mu_{n} \in F,
$$

i.e., $\mathcal{B}$ is a splitting base for $\alpha$ on $V$, and each $\alpha\left(e_{i}\right)=\alpha\left(f_{j}\right)=0$. The $v$-norm $\alpha$ on $V$ induces the $v$-gauge $\operatorname{End}(\alpha)$ on $\operatorname{End}_{D}(V)$. For $g \in \operatorname{End}_{D}(V)$, if $g$ has matrix $\left(c_{i j}\right)$ relative to $\mathcal{B}$, then $\operatorname{End}(\alpha)(g)=\min _{1 \leq i, j \leq n}\left(v\left(c_{i j}\right)\right)$. The matrix for $\sigma(g)$ has the same set of entries up to sign as $\left(c_{i j}\right)$, though the entries are relocated. Hence, $\operatorname{End}(\alpha)$ is invariant under $\sigma$. We exclude this case of $A$ split and $\sigma$ symplectic for the rest of the proof. We may then choose an $F$-linear involution $\theta$ on $D$ of the same type as $\sigma$ and an even hermitian form $h$ on $V$ with respect to $\theta$ such that $\sigma=\operatorname{ad}_{h}$, see [12, (4.2)]. By [16, Cor. 3.6], there exists a $w$-norm $\alpha$ on $V$ that is compatible with $h$. By [18, Prop. 1.19], End $(\alpha)$ is a $v$-gauge; by Proposition 1.7, this gauge is invariant under $\sigma$.

Theorem 2.2 With the hypotheses of this section, if $\sigma$ is anisotropic, then for the Henselian valuation $v$ on $F$ there is a unique $\sigma$-special value function $\varphi$ on A for $v$. This $\varphi$ is a tame $v$-gauge and its value set $\Gamma_{A}$ lies in the divisible hull of $\Gamma_{F}$. It is the unique v-gauge on A invariant under $\sigma$.

Proof We use the same notation as in the proof of Proposition 2.1, representing $A=$ $\operatorname{End}_{D}(V)$ as in that proof. Since $\sigma$ is anisotropic, it is not a symplectic involution on a split algebra. Therefore, it is the adjoint involution of some even Hermitian form $h$ on $V$ with respect to an involution $\theta$ on $D$ of the same type as $\sigma$, see [12, Th. (4.2)]. The form $h$ is anisotropic since $\sigma$ is anisotropic. By [16, Th. 4.6 and Prop. 4.2], the map $\alpha: V \rightarrow \frac{1}{2} \Gamma_{D} \cup\{\infty\}$ defined by

$$
\alpha(x)=\frac{1}{2} w(h(x, x))
$$

is a $w$-norm on $V$ that is compatible with $h$, and the residue form $\widetilde{h}$ is aniso-tropic. Proposition 1.7 then shows that $\varphi=\operatorname{End}(\alpha)$ is a surmultiplicative $v$-value function on $A$ that is invariant under $\sigma$, and Proposition 1.8 shows that $\varphi$ is $\sigma$-special. Since $A$ is tame over $F$, the valuation $w$ is a $v$-gauge on $D$ by [18, Prop. 1.13], hence $\varphi$ is a tame $v$-gauge by [18, Prop. 1.19]. Its value set obviously lies in the divisible hull of $\Gamma_{D}$, which is also the divisible hull of $\Gamma_{F}$.

To prove uniqueness, suppose $\varphi_{1}$ and $\varphi_{2}$ are each $\sigma$-special value functions on $A$ for $v$. To show that $\varphi_{1}=\varphi_{2}$, we argue by induction on the matrix size $\operatorname{ms}(A)$, which is defined as the dimension of $V$ in the representation $A=\operatorname{End}_{D}(V)$. 
Suppose first that $A$ is a division algebra. For any subfield $L \subseteq A$ fixed elementwise under $\sigma$ we have

$$
\varphi_{i}\left(x^{2}\right)=\varphi_{i}(\sigma(x) x)=2 \varphi_{i}(x) \text { for all } x \in L \text { and } i=1,2,
$$

hence for nonzero $\xi=\widetilde{x}^{\varphi_{i}} \in \operatorname{gr}_{\varphi_{i}}(L)$ we have $\xi^{2}={\widetilde{x^{2}}}^{\varphi_{i}} \neq 0$. Therefore, $\operatorname{gr}_{\varphi_{i}}(L)$ is semisimple. By [18, Prop. 1.8], it follows that $\varphi_{1}$ and $\varphi_{2}$ coincide with the unique valuation on $L$ extending $v$. (The extension of $v$ to $L$ is unique because $(F, v)$ is Henselian.) For any $x \in A$, the product $\sigma(x) x$ lies in a subfield of $A$ fixed under $\sigma$, so $\varphi_{1}(\sigma(x) x)=\varphi_{2}(\sigma(x) x)$. Therefore,

$$
\varphi_{1}(x)=\frac{1}{2} \varphi_{1}(\sigma(x) x)=\frac{1}{2} \varphi_{2}(\sigma(x) x)=\varphi_{2}(x)
$$

The claim is thus proved if $\operatorname{ms}(A)=1$.

Suppose next that $\operatorname{ms}(A)>1$. We may then find in $A$ a symmetric idempotent $e \neq 0,1$. (Representing $A=\operatorname{End}_{D}(V)$ as above, we have $\operatorname{dim}_{D} V>1$ and we may take for $e$ the orthogonal projection onto any nonzero proper subspace of $V$.) Let $f=$ $1-e$. The involution $\sigma$ restricts to $e A e$ and $f A f$, and $\operatorname{ms}(e A e), \operatorname{ms}(f A f)<\operatorname{ms}(A)$. By the induction hypothesis, the restrictions of $\varphi_{1}$ and $\varphi_{2}$ coincide on $e A e$ and $f A f$. For any $x \in A$, we have $\sigma(x e) x e \in e A e$ and $\sigma(x f) x f \in f A f$, hence

$$
\varphi_{1}(\sigma(x e) x e)=\varphi_{2}(\sigma(x e) x e) \text { and } \varphi_{1}(\sigma(x f) x f)=\varphi_{2}(\sigma(x f) x f) .
$$

Since $\varphi_{1}$ and $\varphi_{2}$ are $\sigma$-special value functions, Proposition 1.1 shows that

$$
\varphi_{1}(x e)=\varphi_{2}(x e) \text { and } \varphi_{1}(x f)=\varphi_{2}(x f) .
$$

On the other hand, we have $x e \sigma(x f)=0$ and $x e+x f=x$, hence Proposition 1.1 also yields

$$
\varphi_{1}(x)=\min \left(\varphi_{1}(x e), \varphi_{1}(x f)\right) \quad \text { and } \quad \varphi_{2}(x)=\min \left(\varphi_{2}(x e), \varphi_{2}(x f)\right) .
$$

By (2.2), it follows that $\varphi_{1}(x)=\varphi_{2}(x)$.

Now, suppose $g$ is a gauge on $A$ that is invariant under $\sigma$. By [18, Th. 3.1] we may find a $w$-norm $\beta$ on $V$ such that $g=\operatorname{End}(\beta)$. Up to the addition of a constant, we may assume $\beta$ is compatible with $h$ in view of Proposition 1.7. But the norm $\alpha$ of (2.1) is the only $w$-norm on $V$ that is compatible with $h$ by [16, Prop. 4.2], so $\beta=\alpha$ and $g=\operatorname{End}(\alpha)$.

If $g$ is a $v$-gauge on $A$ that is invariant under $\sigma$, we denote by $\sigma_{0}$ the 0 -component of $\tilde{\sigma}$. Thus, $\sigma_{0}$ is an involution on the $\bar{F}$-algebra $A_{0}=A_{\geq 0} / A_{>0}$, which may be viewed as the residue algebra of $A$. The algebra $A_{0}$ is semisimple, but not necessarily simple, see [18, Sect. 2]. Note that if $A_{0}=B_{1} \times \cdots \times B_{k}$ with the $B_{i}$ simple, then an involution $\tau$ on $A_{0}$ is anisotropic if and only if $\tau\left(B_{i}\right)=B_{i}$ and $\left.\tau\right|_{B_{i}}$ is anisotropic for $1 \leq i \leq k$. 
Corollary 2.3 With the hypotheses of this section, if $g$ is a v-gauge on A that is invariant under $\sigma$, the following conditions are equivalent:

(a) $\sigma$ is anisotropic;

(b) $\tilde{\sigma}$ is anisotropic;

(c) $\sigma_{0}$ is anisotropic.

Proof The implication (a) $\Rightarrow$ (b) readily follows from Theorem 2.2, and the implications (b) $\Rightarrow$ (a) and (b) $\Rightarrow$ (c) are clear. To prove (c) $\Rightarrow(\mathrm{b})$, suppose $\xi \in \mathrm{gr}_{g}(A)$ is a nonzero homogeneous element such that $\widetilde{\sigma}(\xi) \xi=0$. Every element $\eta \in\left(\xi \operatorname{gr}_{g}(A)\right) \cap A_{0}$ satisfies $\widetilde{\sigma}(\eta) \eta=\sigma_{0}(\eta) \eta=0$. Therefore, $\sigma_{0}$ is isotropic if $\left(\xi \operatorname{gr}_{g}(A)\right) \cap A_{0} \neq\{0\}$. The corollary thus follows from the following general result:

Lemma 2.4 Let A be a graded simple algebra finite-dimensional over a graded field $\mathrm{K}$, and let $\mathrm{I} \subseteq \mathrm{A}$ be a homogeneous right ideal. Then, there is a homogeneous idempotent $e \in \mathrm{A}$ of degree 0 such that $\mathrm{I}=e \mathrm{~A}$.

Proof By [11, Prop. 1.3], we may identify $A=\operatorname{End}_{D}(V)$ for some graded division $\mathrm{K}$-algebra $\mathrm{D}$ and some finite-dimensional graded $\mathrm{D}$-vector space $\mathrm{V}$. Let $\mathrm{W}=$ $\sum \operatorname{im}(f)$, with the sum taken over all homogeneous $f \in \mathrm{I}$. Then, $\mathbf{W}$ is a graded $\mathrm{D}$-subspace of $\mathrm{V}$ and, just as in the ungraded case, $\mathrm{I}=\operatorname{Hom}_{\mathrm{D}}(\mathrm{V}, \mathrm{W})$. Take any graded $\mathrm{D}$-subspace $\mathrm{Y}$ of $\mathrm{V}$, such that $\mathrm{Y}$ is complementary to $\mathrm{W}$, and let $e: \mathrm{V} \rightarrow \mathrm{W}$ be the projection of $\mathrm{V}$ onto $\mathrm{W}$ along $\mathrm{Y}$. Then, the idempotent $e$ is a degree-preserving graded homomorphism, so $e \in \mathrm{A}_{0}$. Clearly, $\mathrm{I}=e \mathrm{~A}$.

Remarks 2.5 (1) In Corollary 2.3, the hypothesis that $(F, v)$ is Henselian is used only to prove that (a) implies (b) and (c); the implications (c) $\Longleftrightarrow$ (b) $\Rightarrow$ (a) hold without this hypothesis (nor any tameness assumption).

(2) Corollary 2.3 may be regarded as a version of Springer's theorem for involutions. In a slightly different form, it has already been proved by Larmour [13, Th. 4.5]: to see this, observe that the residue involutions defined by Larmour are the direct summands of our residue involution $\sigma_{0}$ for a suitable gauge.

If the involution $\sigma$ is isotropic, we may still define up to isomorphism an anisotropic kernel $(A, \sigma)_{\text {an }}$ in such a way that if $A=\operatorname{End}_{D} V$ and $\sigma=\operatorname{ad}_{h}$, then $(A, \sigma)_{\text {an }} \cong$ $\left(\operatorname{End}_{D} V_{0}, \operatorname{ad}_{h_{0}}\right)$ where $\left(V_{0}, h_{0}\right)$ is an anisotropic kernel of $(V, h)$, see [3], and [2] for involutions of the second kind. The same construction holds for graded simple algebras with involution.

Theorem 2.6 Let $\sigma_{1}, \sigma_{2}$ be F-linear involutions on $A$ with $\left.\sigma_{1}\right|_{Z(A)}=\left.\sigma_{2}\right|_{Z(A)}$. For the Henselian valuation $v$ on $F$, let $g_{1}, g_{2}$ be $v$-gauges on $A$ invariant under $\sigma_{1}$ and $\sigma_{2}$ respectively. If $\operatorname{char}(\bar{F})=2$, assume neither $\sigma_{1}$ nor $\sigma_{2}$ is orthogonal. The following conditions are equivalent:

(a) the algebras with involution $\left(A, \sigma_{1}\right)$ and $\left(A, \sigma_{2}\right)$ are isomorphic;

(b) the graded algebras with anisotropic involution $\left(\operatorname{gr}_{g_{1}}(A), \widetilde{\sigma}_{1}\right)_{a n}$ and $\left(\operatorname{gr}_{g_{2}}(A), \widetilde{\sigma}_{2}\right)_{\text {an }}$ are isomorphic. 
Proof It follows from Proposition 1.5 that $\sigma_{i}$ and $\widetilde{\sigma}_{i}$ are of the same type. Therefore, (a) and (b) each imply that $\sigma_{1}$ and $\sigma_{2}$ are of the same type. If $A$ is split and $\sigma_{1}, \sigma_{2}$ are symplectic, then $\operatorname{gr}_{g_{1}}(A)$ and $\operatorname{gr}_{g_{2}}(A)$ are split and $\widetilde{\sigma}_{1}, \widetilde{\sigma}_{2}$ are symplectic, hence hyperbolic. In this case, (a) and (b) both hold trivially. For the rest of the proof, we exclude this case and fix a representation $A=\operatorname{End}_{D} V$ where $V$ is a right vector space over a central division $K$-algebra $D$. We also fix an involution $\theta$ on $D$ of the same type as $\sigma_{1}$ and $\sigma_{2}$, and non-degenerate even hermitian forms $h_{1}, h_{2}$ on $V$ with respect to $\theta$ such that

$$
\sigma_{1}=\operatorname{ad}_{h_{1}} \text { and } \sigma_{2}=\operatorname{ad}_{h_{2}} .
$$

As observed in the proof of Proposition 2.1, the valuation $v$ extends uniquely to a valuation $w$ on $D$. By [18, Th. 3.1] and Proposition 1.7 we may also find norms $\alpha_{1}$ and $\alpha_{2}$ on $V$ that are compatible with $h_{1}$ and $h_{2}$ respectively, such that

$$
g_{1}=\operatorname{End}\left(\alpha_{1}\right) \text { and } g_{2}=\operatorname{End}\left(\alpha_{2}\right)
$$

hence

$$
\operatorname{gr}_{g_{1}}(A)=\operatorname{End}_{\operatorname{gr}(D)}\left(\operatorname{gr}_{\alpha_{1}}(V)\right) \text { and } \operatorname{gr}_{g_{2}}(A)=\operatorname{End}_{\operatorname{gr}(D)}\left(\operatorname{gr}_{\alpha_{2}}(V)\right) .
$$

It then follows from Proposition 1.8 that

$$
\widetilde{\sigma}_{1}=\operatorname{ad}_{\widetilde{h_{1}}} \quad \text { and } \quad \widetilde{\sigma}_{2}=\operatorname{ad}_{\widetilde{h_{2}}}
$$

hence, denoting by $\left(\mathrm{V}_{1}, k_{1}\right)$ and $\left(\mathrm{V}_{2}, k_{2}\right)$ the anisotropic kernels of $\left(\operatorname{gr}_{\alpha_{1}}(V), \widetilde{h_{1}}\right)$ and $\left(\operatorname{gr}_{\alpha_{2}}(V), \widetilde{h_{2}}\right)$ respectively,

$$
\begin{aligned}
& \left(\operatorname{gr}_{g_{1}}(A), \widetilde{\sigma}_{1}\right)_{\mathrm{an}} \cong\left(\operatorname{End}_{\mathrm{gr}(D)}\left(\mathrm{V}_{1}\right), \operatorname{ad}_{k_{1}}\right) \text { and } \\
& \left(\operatorname{gr}_{g_{2}}(A), \widetilde{\sigma}_{2}\right)_{\mathrm{an}} \cong\left(\operatorname{End}_{\operatorname{gr}(D)}\left(\mathrm{V}_{2}\right), \operatorname{ad}_{k_{2}}\right) .
\end{aligned}
$$

If (a) holds, then $h_{1}$ and $h_{2}$ are similar. Scaling $h_{2}$ by a factor in $F^{\times}$, we may assume $h_{1} \cong h_{2}$. By [16, Th. 3.11], the anisotropic kernels of $\widetilde{h_{1}}$ and $\widetilde{h_{2}}$ are isometric, hence (b) holds.

Conversely, if (b) holds, then the anisotropic kernels of $\widetilde{h_{1}}$ and $\widetilde{h_{2}}$ are similar. Scaling $h_{2}$ by a factor in $F^{\times}$, we may assume that they are isometric. By [16, Th. 4.6], it follows that $h_{1}$ and $h_{2}$ are isometric, hence (a) holds.

Corollary 2.7 With the hypotheses of this section, up to Witt-equivalence the graded algebra with involution $\left(\operatorname{gr}_{g}(A), \tilde{\sigma}\right)$ depends only on the Witt-equivalence class of $(A, \sigma)$, and not on the choice of the invariant $v$-gauge $g$.

The results in this section depend heavily on the assumption that $v$ is Henselian. When $v$ is not Henselian, for an anisotropic involution $\sigma$ on $A$ there may be no $\sigma$-special $v$-gauge on $A$, and there may be many $\sigma$-invariant gauges. See Example 6.3 below for an example of this. What happens when $v$ is not Henselian is described in Theorem 6.1. 


\section{Scalar extensions of involutions}

As an application of the results of Sect. 2, we consider a basic case of the problem of determining when an anisotropic involution remains anisotropic over a scalar extension.

Let $\sigma$ be an $F$-linear involution on a finite-dimensional simple algebra $A$ over a field $F$. Assume $v$ is a valuation on $F$ and $A$ carries a $v$-gauge $g$ invariant under $\sigma$. For any extension $\left(L, v_{L}\right)$ of $(F, v)$, we may consider the $v_{L}$-gauge $g \otimes v_{L}$ on the $L$-algebra $A_{L}=A \otimes_{F} L$. By Proposition 1.3, this $v_{L}$-gauge is invariant under the involution $\sigma \otimes \mathrm{id}_{L}$ on $A_{L}$. If the "residue" involution $\left(\sigma \otimes \mathrm{id}_{L}\right)_{0}$ is anisotropic, then $\sigma \otimes \mathrm{id}_{L}$ is anisotropic by Corollary 2.3 and Remark 2.5(1), and the converse holds if $v_{L}$ is Henselian and $A_{L}$ is tame over $L$, unless $\sigma$ is orthogonal and $\operatorname{char}(\bar{F})=2$. We consider below a case where this residue can be explicitly calculated.

We first recall some facts which will be used repeatedly below. Let $\alpha$ be a surmultiplicative $v$-norm on a finite-dimensional algebra $A$ over a field $F$ with valuation $v$. If $e$ is an idempotent of $A$ with $\alpha(e)=0$ and $N$ is any $F$-subspace of $A$, then $\widetilde{e}^{2}=\widetilde{e}$ in $\operatorname{gr}(A)$ and by [18, Lemma 1.7],

$$
\operatorname{gr}(e N)=\widetilde{e} \operatorname{gr}(N) \text { and } \operatorname{gr}(N e)=\operatorname{gr}(N) \widetilde{e} \operatorname{ingr}(A) .
$$

If $e \neq 1$, let $f=1-e$. Then, $\alpha(f) \geq \min (\alpha(1), \alpha(e))=0$, but since $f^{2}=f$, $\alpha(f) \leq 0$. So, $\alpha(f)=0$, hence $\widetilde{f}=\widetilde{1}-\widetilde{e}$ in $\operatorname{gr}(A)$, and hence

$$
\operatorname{gr}(A)=\widetilde{e} \operatorname{gr}(A) \oplus \widetilde{f} \operatorname{gr}(A)=\operatorname{gr}(e A) \oplus \operatorname{gr}(f A)
$$

Therefore, by [16, Remark 2.6], the direct sum decomposition $A=e A \oplus f A$ is a splitting decomposition, i.e., $\alpha(a)=\min (\alpha(e a), \alpha(f a))$ for any $a \in A$. Likewise $A=A e \oplus A f$ is a splitting decomposition.

Recall also that an element $s \in A^{\times}$is said to be $\alpha$-stable if $\alpha\left(s^{-1}\right)=-\alpha(s)$. For such an $s$ we have by [18, Lemma 1.3 and (1.5)], for every $a \in A$,

$$
\alpha(a s)=\alpha(s a)=\alpha(a)+\alpha(s), \text { hence } \tilde{a} \widetilde{s}=\tilde{a s} \text { and } \tilde{s} \tilde{a}=\tilde{s a}
$$

We now make some general observations on the tensor product of valuations. Let $L / F$ be a finite separable field extension. Recall that the separability idempotent of $L$ is the idempotent $e \in L \otimes_{F} L$ determined uniquely by the conditions that

$$
e \cdot(x \otimes 1)=e \cdot(1 \otimes x) \quad \text { for all } x \in L
$$

and the multiplication map $L \otimes_{F} L \rightarrow L$ carries $e$ to 1 , see for instance [12, Prop. (18.10)]. The separability of $L / F$ implies that the bilinear trace form

$$
T: L \times L \rightarrow F, \quad T(x, y)=\operatorname{Tr}_{L / F}(x y)
$$

is nondegenerate. 
Proposition 3.1 Suppose $v: F \rightarrow \Gamma \cup\{\infty\}$ is a valuation that extends uniquely to a valuation $v_{L}$ on $L$, and that the valued field extension $\left(L, v_{L}\right)$ of $(F, v)$ is tame. Then $v_{L}$ is a v-norm on $L$ which is compatible with the bilinear trace form $T$, and $\left(v_{L} \otimes v_{L}\right)(e)=0$.

Proof Since $v_{L}$ is the unique valuation extending $v$ to $L$ and since the extension is defectless, it follows that $v_{L}$ is a $v$-norm (indeed, a $v$-gauge) on $L$, see [18, Cor. 1.9].

We claim that $v\left(\operatorname{Tr}_{L / F}(x)\right) \geq v_{L}(x)$ for all $x \in L^{\times}$. To see this, consider a Galois closure $M$ of $L$ over $F$ and an extension $v_{M}$ of $v$ to $M$. For every $F$-linear embedding $\iota: L \hookrightarrow M$ the composition $v_{M} \circ \iota$ is a valuation on $L$ extending $v$, hence $v_{M} \circ \iota=v_{L}$. Since $\operatorname{Tr}_{L / F}(x)=\sum \iota(x)$, where the sum extends over all embeddings $\iota: L \hookrightarrow M$, we have

$$
v\left(\operatorname{Tr}_{L / F}(x)\right)=v_{M}\left(\sum_{\iota} \iota(x)\right) \geq \min _{\iota}\left(v_{M} \circ \iota(x)\right)=v_{L}(x),
$$

proving the claim. It follows that for all $x, y \in L^{\times}$,

$$
v(T(x, y)) \geq v_{L}(x)+v_{L}(y) .
$$

To show that $v_{L}$ is compatible with $T$, it remains to show that for any $x \in L^{\times}$there exists $y \in L^{\times}$for which equality holds in (3.4). For this, it suffices to show that there exists $\ell \in L^{\times}$such that $v\left(\operatorname{Tr}_{L / F}(\ell)\right)=v_{L}(\ell)$, since equality then holds in (3.4) with $y=\ell x^{-1}$. For every $\ell \in L^{\times}$with $v_{L}(\ell)=0$ we have

$$
\overline{\operatorname{Tr}_{L / F}(\ell)}=\left|\Gamma_{L}: \Gamma_{F}\right| \cdot \operatorname{Tr}_{\bar{L} / \bar{F}}(\bar{\ell})
$$

by [6, p. 65, Cor. 1]. (Ershov assumes his valuation is Henselian; but the result carries over to the situation here: Let $F_{h}$ be the Henselization of $F$ with respect to $v$. Since the unique extension of $v$ to $L$ is defectless, for any compositum of $L$ with $F_{h}$ we have $\left[L \cdot F_{h}: F_{h}\right] \geq[\bar{L}: \bar{F}]\left|\Gamma_{L}: \Gamma_{F}\right|=[L: F]$. Hence, $L \otimes_{F} F_{h}$ is a field, and (3.5) holds for $L / F$ because it holds for $\left(L \otimes_{F} F_{h}\right) / F_{h}$.)

Since $L / F$ is tame, the residue extension $\bar{L} / \bar{F}$ is separable and $\operatorname{char}(\bar{F})$ does not divide $\left|\Gamma_{L}: \Gamma_{F}\right|$. Therefore, we may find $\ell \in L$ such that $v_{L}(\ell)=0$ and $\operatorname{Tr}_{\bar{L}} / \bar{F}(\bar{\ell}) \neq 0$. Then (3.5) shows that $\overline{\operatorname{Tr}_{L / F}(\ell)} \neq 0$, hence

$$
v\left(\operatorname{Tr}_{L / F}(\ell)\right)=0=v_{L}(\ell)
$$

Therefore, $v_{L}$ is compatible with $T$; it thus coincides with its dual norm $v_{L}^{\sharp}$.

To complete the proof, we compute $\left(v_{L} \otimes v_{L}\right)(e)$. Let $\left(\ell_{i}\right)_{i=1}^{n}$ be a splitting $F$-base of $L$ for $v_{L}$, and let $\left(\ell_{i}^{\sharp}\right)_{i=1}^{n}$ be the dual base for the form $T$. By [12, Prop. (18.12)] we have

$$
e=\sum_{i=1}^{n} \ell_{i} \otimes \ell_{i}^{\sharp},
$$


hence,

$$
\left(v_{L} \otimes v_{L}\right)(e)=\min _{1 \leq i \leq n}\left(v_{L}\left(\ell_{i}\right)+v_{L}\left(\ell_{i}^{\sharp}\right)\right) .
$$

Now, for all $i=1, \ldots, n$ we have $v_{L}\left(\ell_{i}^{\sharp}\right)=v_{L}^{\sharp}\left(\ell_{i}^{\sharp}\right)=-v_{L}\left(\ell_{i}\right)$ by [16, Lemma 3.4]. Therefore, $\left(v_{L} \otimes v_{L}\right)(e)=0$.

Continuing with the same notation and hypotheses as in Proposition 3.1, we now assume further that the extension $L / F$ is Galois. Let $G$ denote its Galois group. Since $v_{L}$ is the unique extension of $v$ to $L, v_{L} \circ \iota=v_{L}$ for any $\iota \in G$, and hence $\iota$ induces a $\operatorname{graded} \operatorname{gr}(F)$-automorphism $\tau$ of $\operatorname{gr}(L)$. For $\iota \in G$, let

$$
e_{\iota}=(\mathrm{id} \otimes \iota)(e) \in L \otimes_{F} L
$$

and let $\widetilde{e}_{\iota}$ be the image of $e_{\iota}$ in $\operatorname{gr}\left(L \otimes_{F} L\right)$, which is canonically identified with $\operatorname{gr}(L) \otimes \operatorname{gr}(F) \operatorname{gr}(L)$ by Proposition 1.3.

Lemma 3.2 The elements $\left(e_{\iota}\right)_{\iota \in G}$ form a family of orthogonal idempotents such that $\sum_{\iota \in G} e_{\iota}=1$. They are the primitive idempotents of $L \otimes_{F}$ L. They satisfy $\left(v_{L} \otimes v_{L}\right)\left(e_{\iota}\right)=0$ and

$$
e_{\iota} \cdot(x \otimes 1)=e_{\iota} \cdot(1 \otimes \iota(x)) \quad \text { for } x \in L
$$

Likewise, for any $y \in \operatorname{gr}(L)$,

$$
\tilde{e}_{\iota} \cdot(y \otimes \tilde{1})=\tilde{e}_{\iota} \cdot(\tilde{1} \otimes \widetilde{\imath}(y)) \quad \text { in } \operatorname{gr}(L) \otimes \operatorname{gr}(F) \operatorname{gr}(L) .
$$

Moreover, $(\iota \otimes \iota)(e)=e$ for $\iota \in G$.

Proof Equation (3.3) shows that $e \cdot\left(L \otimes_{F} L\right)=e \cdot(L \otimes 1) \cong L$. Since $L$ is a field, $e$ must be a primitive idempotent. Equation (3.6) readily follows by applying id $\otimes \iota$ to each side of (3.3). For equation (3.7), it suffices to verify the equality when $y$ is homogeneous and nonzero. But then $y=\tilde{x}$ for some nonzero $x \in L$. Both $x \otimes 1$ and $1 \otimes \iota(x)$ are $v_{L} \otimes v_{L}$-stable in $L \otimes_{F} L$, as defined preceding (3.2) above. Hence, using equations (3.2) and (3.6),

$$
\begin{aligned}
\widetilde{e}_{\iota} \cdot(\widetilde{x} \otimes \widetilde{1}) & =\widetilde{e}_{\iota} \cdot(\widetilde{x \otimes 1})=\left[e_{\iota} \cdot(x \otimes 1)\right]^{\sim} \\
& =\left[e_{\iota} \cdot(1 \otimes \iota(x))\right]^{\sim}=\widetilde{e}_{\iota} \cdot(\widetilde{1 \otimes \iota(x)})=\widetilde{e}_{\iota} \cdot(\widetilde{1} \otimes \widetilde{\imath}(\widetilde{x})) .
\end{aligned}
$$

Since $e$ is a primitive idempotent, it is clear that each $e_{\iota}$ is also a primitive idempotent. For $\iota, \kappa \in G$ and $x \in L$, as $L \otimes_{F} L$ is commutative we have

$$
\begin{aligned}
e_{\iota} e_{\kappa} \cdot(1 \otimes[\kappa(x)-\iota(x)]) & =e_{\iota} e_{\kappa} \cdot(1 \otimes \kappa(x))-e_{\kappa} e_{\iota} \cdot(1 \otimes \iota(x)) \\
& =\left(e_{\iota} e_{\kappa}-e_{\kappa} e_{\iota}\right) \cdot(x \otimes 1)=0 .
\end{aligned}
$$


For $\iota \neq \kappa$, if we choose $x \in L$ with $\iota(x) \neq \kappa(x)$, then $1 \otimes[\kappa(x)-\iota(x)]$ is a unit of $L \otimes_{F} L ;$ hence, $e_{\iota} e_{\kappa}=0$.

As observed in the proof of Proposition 3.1, we have $e=\sum_{i=1}^{n} \ell_{i} \otimes \ell_{i}^{\#}$ if $\left(\ell_{i}\right)_{i=1}^{n}$ is an $F$-base of $L$ and $\left(\ell_{i}^{\sharp}\right)_{i=1}^{n}$ is the dual base for the bilinear form $T$. It follows that $e_{\iota}=\sum_{i=1}^{n} \ell_{i} \otimes \iota\left(\ell_{i}^{\sharp}\right)$ for $\iota \in G$, hence

$$
\sum_{\iota \in G} e_{\iota}=\sum_{i=1}^{n} \ell_{i} \otimes \operatorname{Tr}_{L / F}\left(\ell_{i}^{\sharp}\right) .
$$

Since $\left(\ell_{i}^{\sharp}\right)_{i=1}^{n}$ is the dual base of $\left(\ell_{i}\right)_{i=1}^{n}$, we have

$$
x=\sum_{i=1}^{n} \ell_{i} \operatorname{Tr}_{L / F}\left(\ell_{i}^{\sharp} x\right) \quad \text { for } x \in L .
$$

In particular, $\sum_{i=1}^{n} \ell_{i} \operatorname{Tr}_{L / F}\left(\ell_{i}^{\sharp}\right)=1$, and equation (3.8) yields $\sum_{\iota \in G} e_{\iota}=1$. So, the $e_{\iota}$ are all the primitive idempotents of $L \otimes_{F} L$.

Since $v_{L}$ is the unique valuation extending $v$ to $L$, we have $v_{L} \circ \iota=v_{L}$ for all $\iota \in G$, hence

$$
\left(v_{L} \otimes v_{L}\right)\left(e_{\iota}\right)=\left(v_{L} \otimes v_{L}\right)(e)=0 \quad \text { for all } \iota \in G .
$$

Finally, it is clear that $(\iota \otimes \iota)(e)$ satisfies the same equation (3.3) as $e$ and is carried to 1 by the multiplication map $L \otimes_{F} L \rightarrow L$. Since these properties determine $e$ uniquely, we have $(\iota \otimes \iota)(e)=e$ for all $\iota \in G$.

It is well-known (cf. [15, pp. 256-257, Lemma b]) that the primitive idempotents of $L \otimes_{F} L$ are indexed by $G$ and satisfy (3.6). The further properties of the $e_{\iota}$ given in Lemma 3.2 will be useful in what follows.

Now, assume further that $L \subseteq D$ for some finite-dimensional division $F$-algebra $D$, and that $v$ extends to a valuation $v_{D}$ on $D$ such that $D / F$ is defectless; i.e., $v_{D}$ is a $v$-norm on $D$. The restriction of $v_{D}$ to $L$ is then the unique valuation $v_{L}$ extending $v$. We will use the idempotents $\left(e_{\iota}\right)_{\iota \in G}$ to analyze extensions of involutions from $D$ to $D \otimes_{F} L$. Let $C$ be the centralizer $C_{D}(L)$. Viewing $D$ as a right $C$-vector space, we have the canonical isomorphism

$$
\eta: D \otimes_{F} L \stackrel{\sim}{\rightarrow} \operatorname{End}_{C}(D),
$$

which carries $d \otimes \ell$ to the map $x \mapsto d x \ell$ for $d, x \in D$ and $\ell \in L$. For $\iota \in G$, consider the following $C$-subspace of $D$ :

$$
D_{\iota}=\{d \in D \mid \ell d=d \iota(\ell) \text { for all } \ell \in L\} .
$$

Since $\iota$ on $L$ is induced by an inner automorphism of $D$ by Skolem-Noether, $D_{\iota} \neq\{0\}$. Since in addition, $D_{\text {id }}=C$ and $D_{\kappa} \cdot D_{\iota} \subseteq D_{\iota \kappa}$ for all $\iota, \kappa \in G$, we must have $\operatorname{dim}_{C}\left(D_{\iota}\right)=1$ for each $\iota$. 
Lemma 3.3 We have $D \otimes_{F} L=\bigoplus_{\iota \in G} e_{\iota}(D \otimes 1)$ and $D=\bigoplus_{\iota \in G} D_{l}$, and these direct sums are splitting decompositions of $D \otimes_{F} L$ and $D$ with respect to $v_{D} \otimes v_{L}$ and $v_{D}$, respectively. More precisely, we have

$$
\left(v_{D} \otimes v_{L}\right)\left(\sum_{\iota \in G} e_{\iota} \cdot\left(x_{\iota} \otimes 1\right)\right)=\min _{\iota \in G}\left(v_{D}\left(x_{\iota}\right)\right) \quad \text { for } x_{\iota} \in D,
$$

and

$$
v_{D}\left(\sum_{\iota \in G} y_{\iota}\right)=\min _{\iota \in G}\left(v_{D}\left(y_{\iota}\right)\right) \quad \text { for } y_{\iota} \in D_{\iota}
$$

Furthermore, for all $\iota, \kappa \in G$,

$$
e_{\iota}\left(D \otimes_{F} L\right) e_{\kappa}=e_{\iota}\left(D_{\kappa^{-1} \iota} \otimes 1\right)
$$

Proof Let $A=D \otimes_{F} L$ and $\alpha=v_{D} \otimes v_{L}$. Since $\left(e_{\iota}\right)_{\iota \in G}$ is a family of orthogonal idempotents with $\sum_{\iota \in G} e_{\iota}=1$ and $\alpha\left(e_{\iota}\right)=0$ for each $\iota$, the collection $\left(\widetilde{e}_{\iota}\right)_{\iota \in G}$ is a family of orthogonal idempotents in $\operatorname{gr}(A)$ with $\sum_{l \in G} \widetilde{e}_{\iota}=\widetilde{1}$. Hence, using (3.1),

$$
A=\bigoplus_{\iota \in G} e_{\iota} A \quad \text { and } \operatorname{gr}(A)=\bigoplus_{\iota \in G} \tilde{e}_{\iota} \operatorname{gr}(A)=\bigoplus_{\iota \in G} \operatorname{gr}\left(e_{\iota} A\right) .
$$

Likewise, for any $\iota \in G$,

$$
e_{\iota} A=\bigoplus_{\kappa \in G} e_{\iota} A e_{\kappa} \quad \text { and } \operatorname{gr}\left(e_{\iota} A\right)=\bigoplus_{\kappa \in G} \operatorname{gr}\left(e_{\iota} A\right) \widetilde{e}_{\kappa}=\bigoplus_{\kappa \in G} \operatorname{gr}\left(e_{\iota} A e_{\kappa}\right)
$$

In view of (3.6), we have $e_{\iota} \cdot(1 \otimes L)=e_{\iota} \cdot(L \otimes 1)$, hence $e_{\iota} \cdot\left(D \otimes_{F} L\right)=e_{\iota} \cdot(D \otimes 1)$. For any nonzero $x \in D, \alpha(x \otimes 1)=v_{D}(x)$ and

$$
\alpha\left((x \otimes 1)^{-1}\right)=\alpha\left(x^{-1} \otimes 1\right)=-v_{D}(x) .
$$

So, $x \otimes 1$ is $\alpha$-stable, and (3.2) applies. Since (3.10) shows that the direct sum $A=$ $\bigoplus_{\iota \in G} e_{\iota} A$ is a splitting decomposition of $A$ for $\alpha$, it follows using (3.2) that for any $x_{\iota} \in D$,

$$
\begin{aligned}
\alpha\left(\sum_{\iota \in G} e_{\iota} \cdot\left(x_{\iota} \otimes 1\right)\right) & =\min _{\iota \in G}\left(\alpha\left(e_{\iota} \cdot\left(x_{\iota} \otimes 1\right)\right)\right) \\
& =\min _{\iota \in G}\left(\alpha\left(e_{\iota}\right)+\alpha\left(x_{\iota} \otimes 1\right)\right)=\min _{\iota \in G}\left(v_{D}\left(x_{\iota}\right)\right) .
\end{aligned}
$$

To prove the rest, we use the canonical isomorphism $\eta$ of (3.9). For each $\iota \in G$, let $\pi_{\iota}=\eta\left(e_{\iota}\right)$, which is a projection in $\operatorname{End}_{C}(D)$. By (3.6) and the commutativity of $L \otimes_{F} L$, for any $\ell \in L$ and $d \in D$,

$$
\ell \cdot \pi_{\iota}(d)=\eta\left((\ell \otimes 1) e_{\iota}\right)(d)=\eta\left((1 \otimes \iota(\ell)) e_{\iota}\right)(d)=\pi_{\iota}(d) \cdot \iota(\ell) .
$$


Hence, $\pi_{\iota}(D) \subseteq D_{\iota}$. Since $\operatorname{im}\left(\pi_{\iota}\right)$ is a nonzero $C$-subspace of the 1-dimensional $C$-vector space $D_{\iota}$, it follows that $\operatorname{im}\left(\pi_{\iota}\right)=D_{\iota}$. Because $\left(\pi_{\iota}\right)_{\iota \in G}$ is a family of orthogonal idempotents of $\operatorname{End}_{C}(D)$ such that $\sum_{\iota \in G} \pi_{\iota}=\operatorname{id}_{D}$, we have $D=\bigoplus_{\iota \in G} D_{\iota}$; furthermore, each $\pi_{\iota}$ is the projection of $D$ onto $D_{\iota}$ parallel to $\bigoplus_{\kappa \neq \iota} D_{\kappa}$. Thus, for any $\iota, \kappa \in G, \pi_{\iota} \operatorname{End}_{C}(D) \pi_{\kappa}$ consists of those endomorphisms sending $D_{\kappa}$ to $D_{\iota}$ and $D_{\lambda}$ to $\{0\}$ for $\lambda \neq \kappa$. For any $\lambda \in G$, since $D_{\kappa^{-1} \iota} D_{\lambda} \subseteq D_{\lambda \kappa^{-1} \iota}$, we have

$$
\left[\pi_{\iota} \circ \eta\left(D_{\kappa^{-1} \iota} \otimes 1\right)\right]\left(D_{\lambda}\right)=\pi_{\iota}\left(D_{\kappa^{-1} \iota} D_{\lambda}\right) \subseteq \pi_{\iota}\left(D_{\lambda \kappa^{-1} \iota}\right) \subseteq \begin{cases}D_{\iota}, & \text { if } \lambda=\kappa \\ \{0\}, & \text { if } \lambda \neq \kappa\end{cases}
$$

Hence, $\pi_{\iota} \circ \eta\left(D_{\kappa^{-1} \iota} \otimes 1\right) \subseteq \pi_{\iota} \operatorname{End}_{C}(D) \pi_{\kappa}$. By applying $\eta^{-1}$, this yields

$$
e_{\iota}\left(D_{\kappa^{-1} \iota} \otimes 1\right) \subseteq e_{\iota} A e_{\kappa} \text { for all } \iota, \kappa \in G
$$

Now, fix $\iota \in G$. We have seen that $e_{\iota} A=e_{\iota}(D \otimes 1)$. The $F$-epimorphism $\rho_{\iota}: D \rightarrow$ $e_{\iota} A$ given by $d \mapsto e_{\iota}(d \otimes 1)$ is clearly injective; $\rho_{\iota}$ is also norm-preserving, as $\alpha\left(e_{\iota}\right)=0$ and $d \otimes 1$ is stable in $A$ for each nonzero $d \in D$. Since $D=\bigoplus_{\kappa \in G} D_{\kappa^{-1}}$, we have

$$
\begin{aligned}
\bigoplus_{\kappa \in G} e_{\iota} A e_{\kappa} & =e_{\iota} A=\rho_{\iota}(D)=\bigoplus_{\kappa \in G} \rho_{\iota}\left(D_{\kappa^{-1} \iota}\right) \\
& =\bigoplus_{\kappa \in G} e_{\iota}\left(D_{\kappa^{-1} \iota} \otimes 1\right) .
\end{aligned}
$$

This shows that the inclusions in (3.12) must all be equalities. It follows from (3.11) above that the direct sum decomposition $\bigoplus_{\kappa \in G} e_{\iota} A e_{\kappa}$ is a splitting decomposition of $e_{\iota} A$. Therefore, by applying the norm-preserving map $\rho_{\iota}^{-1}$ to the terms in (3.13), it follows that $\bigoplus_{\kappa \in G} D_{\kappa}$ is a splitting decomposition of $D$.

While $D \otimes_{F} L$ is simple, the degree 0 part $\left(D \otimes_{F} L\right)_{0}$ of $\operatorname{gr}\left(D \otimes_{F} L\right)$ is in general only semisimple. The value sets $\Gamma_{D_{\iota}}$ of the $D_{\iota}$ encode how $\left(D \otimes_{F} L\right)_{0}$ decomposes: Since each $D_{\iota}$ is a 1-dimensional $C$-subspace of $D$ and $\left.v_{D}\right|_{D_{\iota}}$ is a $\left.v_{D}\right|_{C}$-norm on $D_{\iota}$, each $\Gamma_{D_{\iota}}$ is a coset of $\Gamma_{C}$ in $\Gamma_{D}$. Therefore, there is a well-defined map

$$
\psi: G \rightarrow \Gamma_{D} / \Gamma_{C} \text { given by } \psi(\iota)=\Gamma_{D_{\iota}} .
$$

Because $D_{\iota} \cdot D_{\kappa} \subseteq D_{\kappa \iota}$ and $\Gamma_{D}$ is abelian, $\psi$ is a group homomorphism, which is surjective since $D=\bigoplus_{\iota \in G} D_{\iota}$ is a splitting decomposition of $D$ by Lemma 3.3. So, $|\operatorname{ker}(\psi)|=|G| /\left|\Gamma_{D}: \Gamma_{C}\right|=[D: C] /\left|\Gamma_{D}: \Gamma_{C}\right|$, which shows that

$\psi$ is injective if and only if $D$ is totally ramified over $C$.

Lemma 3.4 Let $A=D \otimes_{F}$ L. Then, each $\widetilde{e}_{\iota}$ is a primitive idempotent of $A_{0}$, and $A_{0}=\bigoplus_{\iota \in G} \widetilde{e}_{\iota} A_{0}=\bigoplus_{\iota \in G} \widetilde{e}_{\iota}\left(D_{0} \otimes 1\right)$. For any $\iota, \kappa \in G$,

$$
\widetilde{e}_{\iota} A_{0} \widetilde{e}_{\kappa}=\left\{\begin{array}{cl}
\widetilde{e}_{\iota}\left(\left(D_{\kappa^{-1} \iota_{\iota}}\right)_{0} \otimes 1\right) \neq 0, & \text { if } \psi(\kappa)=\psi(\iota) \\
0, & \text { if } \psi(\kappa) \neq \psi(\iota)
\end{array}\right.
$$


Proof We saw in (3.10) that $\operatorname{gr}(A)=\bigoplus_{\iota \in G} \widetilde{e}_{\iota} \operatorname{gr}(A)$. Moreover, as $\operatorname{gr}(A)=$ $\operatorname{gr}(D) \otimes \operatorname{gr}(F) \operatorname{gr}(L)$ and $\widetilde{e}_{\iota}(1 \otimes \operatorname{gr}(L))=\widetilde{e}_{\iota}(\operatorname{gr}(L) \otimes 1)$ by (3.7), we have $\widetilde{e}_{\iota} \operatorname{gr}(A)=\widetilde{e}_{\iota}(\operatorname{gr}(D) \otimes 1)$. So, for the degree 0 components we have $A_{0}=$ $\bigoplus_{\iota \in G} \widetilde{e}_{\iota} A_{0}=\bigoplus_{\iota \in G} \widetilde{e}_{\iota}\left(D_{0} \otimes 1\right)$. Similarly, for $\iota, \kappa \in G$, by (3.1) and Lemma 3.3,

$$
\begin{aligned}
\widetilde{e}_{\iota} \operatorname{gr}(A) \widetilde{e}_{\kappa} & =\operatorname{gr}\left(e_{\iota} A e_{\kappa}\right)=\operatorname{gr}\left(e_{\iota}\left(D_{\kappa^{-1} \iota} \otimes 1\right)\right) \\
& =\widetilde{e}_{\iota} \operatorname{gr}\left(D_{\kappa^{-1} \iota} \otimes 1\right)=\widetilde{e}_{\iota}\left(\operatorname{gr}\left(D_{\kappa^{-1} \iota}\right) \otimes 1\right)
\end{aligned}
$$

Hence, for the degree 0 components,

$$
\widetilde{e}_{\iota} A_{0} \widetilde{e}_{\kappa}=\widetilde{e}_{\iota}\left(\left(D_{\kappa^{-1} \iota}\right)_{0} \otimes 1\right)
$$

If $\psi(\kappa) \neq \psi(\iota)$, then $\psi\left(\kappa^{-1} \iota\right)$ is a nonzero element of $\Gamma_{D} / \Gamma_{C}$, so $\left(D_{\kappa^{-1}}\right)_{0}=\{0\}$. If $\psi(\kappa)=\psi(\iota)$, then $\left(D_{\kappa^{-1} \iota}\right)_{0} \neq 0$, and since nonzero elements of $D_{\kappa^{-1} \iota} \otimes 1$ are stable, (3.2) yields $\widetilde{e}_{\iota}\left(\left(D_{\kappa^{-1}}\right)_{0} \otimes 1\right) \neq\{0\}$. If $\kappa=\iota$, then $\left(D_{\kappa^{-1} \iota}\right)_{0} \otimes 1=C_{0} \otimes 1$, so $\widetilde{e}_{\iota} A_{0} \widetilde{e}_{\iota}=\widetilde{e}_{\iota}\left(C_{0} \otimes 1\right)$. Since $C \otimes 1$ centralizes $e_{\iota} \in L \otimes_{F} L, C_{0} \otimes 1$ centralizes $\widetilde{e}_{\iota}$. Hence, $\widetilde{e}_{l} A_{0} \widetilde{e}_{l} \cong C_{0} \otimes 1 \cong C_{0}$. Since $C_{0}$ is a division ring, $\widetilde{e}_{l}$ is a primitive idempotent of $A_{0}$.

Now, assume $\sigma$ is an $F$-linear involution on $D$ which stabilizes $L$, and therefore restricts to an automorphism $\sigma_{L}$ of $L$, and let $\iota \in G$ be such that $\iota^{2}=$ id. Then $\sigma \otimes \iota$ is an involution on $D \otimes_{F} L$. Since the valuation $v_{D}$ extending $v$ to $D$ is unique by [19, Th.], it is invariant under $\sigma$. Likewise, $v_{L}$ is invariant under $\iota$, hence $v_{D} \otimes v_{L}$ is invariant under $\sigma \otimes \iota$ by Proposition 1.3.

Proposition 3.5 The involution $\sigma \otimes \iota$ on $D \otimes_{F}$ L is isotropic unless $\sigma_{L}=\iota$ and ८ lies in the center $Z(G)$ of $G$. If $\sigma_{L}=\iota \in Z(G)$ and $D$ is totally ramified over $C_{D}(L)$, then $\sigma \otimes \iota$ is anisotropic.

Proof For $\kappa \in G$ we have

$$
\left(\sigma_{L} \otimes \iota\right)\left(e_{\kappa}\right)=\left(\sigma_{L} \otimes \iota \kappa\right)(e)=\left(\operatorname{id}_{L} \otimes \iota \kappa \sigma_{L}\right) \circ\left(\sigma_{L} \otimes \sigma_{L}\right)(e)
$$

Since $\left(\sigma_{L} \otimes \sigma_{L}\right)(e)=e$ by Lemma 3.2, it follows that

$$
\left(\sigma_{L} \otimes \iota\right)\left(e_{\kappa}\right)=e_{\iota \kappa \sigma_{L}}
$$

If $\iota \neq \sigma_{L}$ or if $\iota=\sigma_{L}$ and $\iota \notin Z(G)$, we may find $\kappa \in G$ such that $\iota \kappa \sigma_{L} \neq \kappa$, hence

$$
(\sigma \otimes \iota)\left(e_{\kappa}\right) \cdot e_{\kappa}=e_{\iota \kappa \sigma_{L}} \cdot e_{\kappa}=0
$$

Therefore, $\sigma \otimes \iota$ is isotropic.

Now assume $\sigma_{L}=\iota$ and $\iota \in Z(G)$. So, $(\sigma \otimes \imath)\left(e_{\kappa}\right)=e_{\kappa}$ for all $\kappa \in G$; hence, in $\left(D \otimes_{F} L\right)_{0},(\sigma \otimes \iota)_{0}\left(\widetilde{e}_{\kappa}\right)=\widetilde{e}_{\kappa}$. Assume further that $D$ is totally ramified over 
$C=C_{D}(L)$. Then $\psi$ is injective by (3.14), so by Lemma 3.4, $\widetilde{e}_{l}\left(D \otimes_{F} L\right)_{0} \widetilde{e}_{\kappa}=0$ whenever $\kappa \neq \iota$. Hence,

$$
\left(D \otimes_{F} L\right)_{0}=\bigoplus_{\iota \in G} \widetilde{e}_{\iota}\left(D \otimes_{F} L\right)_{0} \widetilde{e}_{\iota}
$$

Since $(\sigma \otimes \imath)_{0}$ maps each direct summand to itself and each summand is a division ring, $(\sigma \otimes \iota)_{0}$ is anisotropic. It follows from Corollary 2.3 (see also Remark 2.5(1)) that $\sigma \otimes \iota$ is anisotropic.

Corollary 3.6 Let $D$ be a central division algebra over a field $F$. Assume $v$ is a valuation on $F$ which extends to a valuation on $D$ so that $D$ is tame over $F$. Let $\sigma$ be an involution of the first kind on $D$ and let $L \subseteq D$ be a subfield Galois over $F$, consisting of $\sigma$-symmetric elements. If $D$ is totally ramified over $C_{D}(L)$, then the involution $\sigma \otimes \mathrm{id}_{L}$ on $D \otimes_{F} L$ is anisotropic.

Proof This is immediate from Proposition 3.5.

Remarks 3.7 (a) The assumption in Corollary 3.6 that $D$ is totally ramified over $C_{D}(L)$ holds whenever $D$ is totally ramified over $F$. In this case we do not have to assume that $L$ is Galois over $F$. For, since $v$ extends to $D$, it follows from a theorem of Morandi [14] that $D$ remains a division ring after scalar extension to a Henselization $F_{h}$ of $F$ for $v$. Therefore, we may assume that $F$ is Henselian. The extension $L / F$ is then Galois, since it is tame and totally ramified.

(b) Another case in which $D$ is totally ramified over $C_{D}(L)$ occurs whenever the subfield $L$ of $D$ is unramified over $F$ and $\bar{L} \subseteq Z(\bar{D})$.

(c) Another way to obtain the information about $\left(D \otimes_{F} L\right)_{0}$ needed in the proof of Proposition 3.5 is to prove that if the $F$-central division ring $D$ has a valuation tame over $F$ and $L$ is any subfield of $D$ containing $F$, and $C=C_{D}(L)$, then the canonical isomorphism $D \otimes_{F} L \cong \operatorname{End}_{C}(D)$ is norm-preserving; so this induces a graded isomorphism

$$
\operatorname{gr}\left(D \otimes_{F} L\right) \cong \operatorname{gr}\left(\operatorname{End}_{C}(D)\right) \cong \operatorname{End}_{\operatorname{gr}(C)}(\operatorname{gr}(D))
$$

\section{Composition of value functions}

Let $v: F \rightarrow \Gamma \cup\{\infty\}$ be a valuation on a field $F$, and let $\Delta \subset \Gamma$ be a convex subgroup, i.e., if $0 \leq \gamma \leq \delta$ with $\gamma \in \Gamma$ and $\delta \in \Delta$, then $\gamma \in \Delta$. Let $\Lambda=\Gamma / \Delta$, and let $\varepsilon: \Gamma \rightarrow \Lambda$ be the canonical map. The ordering on $\Gamma$ induces a total ordering on $\Lambda$ such that for $\gamma_{1}, \gamma_{2} \in \Gamma$, if $\gamma_{1} \leq \gamma_{2}$, then $\varepsilon\left(\gamma_{1}\right) \leq \varepsilon\left(\gamma_{2}\right)$. Consequently,

$$
\text { if } \varepsilon\left(\gamma_{2}\right)<\varepsilon\left(\gamma_{1}\right), \text { then } \gamma_{2}<\gamma_{1} \text {. }
$$

Because $\Gamma$ is assumed to be divisible, $\Delta$ and $\Lambda$ are also divisible. By composing $v$ with $\varepsilon$, we obtain a coarser valuation on $F$,

$$
w=\varepsilon \circ v: F \rightarrow \Lambda \cup\{\infty\}
$$


Let $\bar{F}^{v}$ (resp. $\bar{F}^{w}$ ) denote the residue field of $F$ for the valuation $v$ (resp. w). The valuation $v$ induces a valuation

$$
u: \bar{F}^{w} \rightarrow \Delta \cup\{\infty\}
$$

with residue field

$$
\overline{\bar{F}}^{w}=\bar{F}^{v},
$$

see [9, pp. 44-45].

Now, let $V$ be an $F$-vector space and let $\alpha: V \rightarrow \Gamma \cup\{\infty\}$ be a $v$-value function. Composition with $\varepsilon$ yields a $w$-value function

$$
\beta=\varepsilon \circ \alpha: V \rightarrow \Lambda \cup\{\infty\}
$$

Each $\lambda \in \Lambda=\Gamma / \Delta$ is a coset of $\Delta$, and may therefore be viewed as a subset of $\Gamma$. For $x \in V$, we have by definition

$$
\beta(x)=\lambda \in \Lambda \text { if and only if } \alpha(x) \in \lambda \subset \Gamma \text {. }
$$

For $\lambda \in \Lambda$, let

$$
\begin{gathered}
V_{\geq \lambda}^{\beta}=\{x \in V \mid \beta(x) \geq \lambda\}, \quad V_{>\lambda}^{\beta}=\{x \in V \mid \beta(x)>\lambda\}, \\
\text { and } V_{\lambda}^{\beta}=V_{\geq \lambda}^{\beta} / V_{>\lambda}^{\beta} .
\end{gathered}
$$

The group $V_{\lambda}^{\beta}$ is an $\bar{F}^{w}$-vector space.

Lemma 4.1 If $x, y \in V_{\geq \lambda}^{\beta}$ satisfy $x \equiv y \not \equiv 0\left(\bmod V_{>\lambda}^{\beta}\right)$, then $\alpha(x)=\alpha(y)$.

Proof We have $\beta(x-y)>\lambda=\beta(y)$. Since $\beta=\varepsilon \circ \alpha$, (4.1) shows that $\alpha(x-y)>$ $\alpha(y)$. Hence, $\alpha(x)=\min (\alpha(x-y), \alpha(y))=\alpha(y)$.

In view of this lemma, we may define

$$
\alpha_{\lambda}: V_{\lambda}^{\beta} \rightarrow \lambda \cup\{\infty\} \quad \text { by } x+V_{>\lambda}^{\beta} \mapsto \begin{cases}\alpha(x) & \text { if } \beta(x)=\lambda \\ \infty & \text { if } \beta(x)>\lambda\end{cases}
$$

Clearly, $\alpha_{\lambda}$ is a $u$-value function on $V_{\lambda}^{\beta}$. For $\gamma \in \lambda$ we have

$$
\left(V_{\lambda}^{\beta}\right)_{\gamma}^{\alpha_{\lambda}}=V_{\gamma}^{\alpha}
$$

Therefore,

$$
\operatorname{gr}_{\alpha}(V)=\bigoplus_{\lambda \in \Lambda} \operatorname{gr}_{\alpha_{\lambda}}\left(V_{\lambda}^{\beta}\right) \quad \text { where } \operatorname{gr}_{\alpha_{\lambda}}\left(V_{\lambda}^{\beta}\right)=\bigoplus_{\gamma \in \lambda} V_{\gamma}^{\alpha}
$$


while

$$
\operatorname{gr}_{\beta}(V)=\bigoplus_{\lambda \in \Lambda} V_{\lambda}^{\beta}
$$

Now, let

$$
\begin{aligned}
& \Gamma_{F}=v\left(F^{\times}\right) \subseteq \Gamma, \quad \Delta_{F}=\Delta \cap \Gamma_{F} \subseteq \Delta, \\
& \Lambda_{F}=w\left(F^{\times}\right)=\Gamma_{F} / \Delta_{F} \subseteq \Lambda .
\end{aligned}
$$

These groups are the value groups of, respectively, $v, u$, and $w$. Similarly, let

$$
\Gamma_{V}=\alpha(V \backslash\{0\}) \subseteq \Gamma \text { and } \Lambda_{V}=\beta(V \backslash\{0\}) \subseteq \Lambda
$$

For each $\lambda \in \Lambda_{V}$, let also

$$
\lambda_{V}=\alpha_{\lambda}\left(V_{\lambda}^{\beta} \backslash\{0\}\right) \subseteq \lambda
$$

Clearly, $\lambda_{V}=\lambda \cap \Gamma_{V}$. Note $\Gamma_{F}$ (resp. $\Delta_{F}$, resp. $\Lambda_{F}$ ) is a subgroup of $\Gamma$ (resp. $\Delta$, resp. $\Lambda$ ), while $\Gamma_{V}$ (resp. $\Lambda_{V}$, resp. $\lambda_{V}$ for $\lambda \in \Lambda_{V}$ ) is a union of cosets of $\Gamma_{F}$ (resp. $\Lambda_{F}$, resp. $\Delta_{F}$ ). We denote by $\left|\Gamma_{V}: \Gamma_{F}\right|$ the cardinality of the set of cosets of $\Gamma_{F}$ in $\Gamma_{V}$, and define likewise $\left|\Lambda_{V}: \Lambda_{F}\right|$ and $\left|\lambda_{V}: \Delta_{F}\right|$ for $\lambda \in \Lambda_{V}$.

Lemma 4.2 If $\operatorname{dim}_{F} V$ is finite, then $\left|\Gamma_{V}: \Gamma_{F}\right|,\left|\Lambda_{V}: \Lambda_{F}\right|$, and $\left|\lambda_{V}: \Delta_{F}\right|$ for $\lambda \in \Lambda_{V}$ are finite. If $\lambda_{1}, \ldots, \lambda_{r} \in \Lambda_{V}$ are representatives of the various cosets of $\Lambda_{V}$ modulo $\Lambda_{F}$, then

$$
\left|\Gamma_{V}: \Gamma_{F}\right|=\sum_{i=1}^{r}\left|\left(\lambda_{i}\right)_{V}: \Delta_{F}\right| .
$$

Proof By [16, Prop. 2.2] we have

$$
\left|\Gamma_{V}: \Gamma_{F}\right| \leq \operatorname{dim}_{F} V, \quad\left|\Lambda_{V}: \Lambda_{F}\right| \leq \operatorname{dim}_{F} V
$$

and also

$$
\left|\lambda_{V}: \Delta_{F}\right| \leq \operatorname{dim}_{\bar{F}} w V_{\lambda}^{\beta} \leq \operatorname{dim}_{F} V \quad \text { for } \lambda \in \Lambda_{V}
$$

For $i=1, \ldots, r$, let $\gamma_{i 1}, \ldots, \gamma_{i s_{i}} \in\left(\lambda_{i}\right)_{V} \subseteq \Gamma$ be representatives of the various cosets of $\left(\lambda_{i}\right)_{V}$ modulo $\Delta_{F}$. Thus,

$$
\left(\lambda_{i}\right)_{V}=\coprod_{j=1}^{s_{i}}\left(\gamma_{i j}+\Delta_{F}\right),
$$

where $\coprod$ denotes the disjoint union. For $\gamma \in \Gamma_{V}$, we have $\varepsilon(\gamma) \in \Lambda_{V}$, hence

$$
\varepsilon(\gamma)=\lambda_{i}+w(a) \text { for some } i \in\{1, \ldots, r\} \text { and some } a \in F^{\times} .
$$


It follows that $\gamma-v(a) \in\left(\lambda_{i}\right)_{V}$; hence,

$$
\gamma-v(a)=\gamma_{i j}+v(b) \text { for some } j \in\left\{1, \ldots, s_{i}\right\} \text { and some } b \in F^{\times} \text {. }
$$

This shows that $\gamma \equiv \gamma_{i j} \bmod \Gamma_{F}$, hence

$$
\Gamma_{V}=\bigcup_{i=1}^{r} \bigcup_{j=1}^{s_{i}}\left(\gamma_{i j}+\Gamma_{F}\right)
$$

To complete the proof, it suffices to show the union is disjoint. If $\gamma_{i j} \equiv \gamma_{k \ell}\left(\bmod \Gamma_{F}\right)$ for some $i, j, k, \ell$, then $\varepsilon\left(\gamma_{i j}\right) \equiv \varepsilon\left(\gamma_{k \ell}\right)\left(\bmod \Lambda_{F}\right)$, hence $i=k$ since $\varepsilon\left(\gamma_{i j}\right)=\lambda_{i}$ and $\varepsilon\left(\gamma_{k \ell}\right)=\lambda_{k}$. Moreover, from $\varepsilon\left(\gamma_{i j}\right)=\varepsilon\left(\gamma_{k \ell}\right)$ it follows that $\gamma_{i j}-\gamma_{k \ell} \in \Delta$, hence $\gamma_{i j} \equiv \gamma_{k \ell}\left(\bmod \Gamma_{F}\right)$ implies $\gamma_{i j} \equiv \gamma_{k \ell}\left(\bmod \Delta_{F}\right)$, hence also $j=\ell$.

Proposition 4.3 Suppose $\operatorname{dim}_{F} V$ is finite, and let $\lambda_{1}, \ldots, \lambda_{r} \in \Lambda_{V}$ be representatives of the various cosets of $\Lambda_{V}$ modulo $\Lambda_{F}$. The following conditions are equivalent:

(i) $\alpha$ is a norm;

(ii) $\beta$ is a norm and $\alpha_{\lambda}$ is a norm for all $\lambda \in \Lambda_{V}$;

(iii) $\beta$ is a norm and $\alpha_{\lambda_{i}}$ is a norm for $i=1, \ldots, r$.

Proof Use the same notation as in the lemma. For simplicity, denote $\alpha_{i}=\alpha_{\lambda_{i}}$ and $V_{i}=V_{\lambda_{i}}^{\beta}$ for $i=1, \ldots, r$, and $V_{i j}=V_{\gamma_{i j}}^{\alpha}$ for $i=1, \ldots, r$ and $j=1, \ldots, s_{i}$, and use the notation $[V: F]$ for $\operatorname{dim}_{F} V$. From (4.2) it follows that

$$
\left[\operatorname{gr}_{\alpha}(V): \operatorname{gr}_{v}(F)\right]=\sum_{i=1}^{r} \sum_{j=1}^{s_{i}}\left[V_{i j}: \bar{F}^{v}\right]
$$

Likewise,

$$
\left[\operatorname{gr}_{\beta}(V): \operatorname{gr}_{w}(F)\right]=\sum_{i=1}^{r}\left[V_{i}: \bar{F}^{w}\right]
$$

and

$$
\left[\operatorname{gr}_{\alpha_{i}}\left(V_{i}\right): \operatorname{gr}_{u}\left(\bar{F}^{w}\right)\right]=\sum_{j=1}^{s_{i}}\left[V_{i j}: \bar{F}^{v}\right] \quad \text { for } i=1, \ldots, r
$$

If $\alpha_{k}$ is not a norm for some $k \in\{1, \ldots, r\}$, then

$$
\left[V_{k}: \bar{F}^{w}\right]>\left[\operatorname{gr}_{\alpha_{k}}\left(V_{k}\right): \operatorname{gr}_{u}\left(\bar{F}^{w}\right)\right]
$$

On the other hand, we have

$$
\left[V_{i}: \bar{F}^{w}\right] \geq\left[\operatorname{gr}_{\alpha_{i}}\left(V_{i}\right): \operatorname{gr}_{u}\left(\bar{F}^{w}\right)\right] \text { for all } i
$$


hence, by (4.4) and (4.5),

$$
\left[\operatorname{gr}_{\beta}(V): \operatorname{gr}_{w}(F)\right]>\sum_{i=1}^{r}\left[\operatorname{gr}_{\alpha_{i}}\left(V_{i}\right): \operatorname{gr}_{u}\left(\bar{F}^{w}\right)\right]=\sum_{i=1}^{r} \sum_{j=1}^{s_{i}}\left[V_{i j}: \bar{F}^{v}\right]
$$

In view of (4.3), it follows that $\left[\operatorname{gr}_{\beta}(V): \operatorname{gr}_{w}(F)\right]>\left[\operatorname{gr}_{\alpha}(V): \operatorname{gr}_{v}(F)\right]$. Since $[V: F] \geq\left[\operatorname{gr}_{\beta}(V): \operatorname{gr}_{w}(F)\right]$, we have $[V: F]>\left[\operatorname{gr}_{\alpha}(V): \operatorname{gr}_{v}(F)\right]$, hence $\alpha$ is not a norm.

If each $\alpha_{i}$ is a norm, then $\left[V_{i}: \bar{F}^{w}\right]=\left[\operatorname{gr}_{\alpha_{i}}\left(V_{i}\right): \operatorname{gr}_{u}(F)\right]$ for $i=1, \ldots, r$, hence (4.4), (4.5), and (4.3) yield

$$
\left[\operatorname{gr}_{\beta}(V): \operatorname{gr}_{w}(F)\right]=\sum_{i=1}^{r} \sum_{j=1}^{s_{i}}\left[V_{i j}: \bar{F}^{v}\right]=\left[\operatorname{gr}_{\alpha}(V): \operatorname{gr}_{v}(F)\right]
$$

It follows that $\alpha$ is a norm if and only if $\beta$ is a norm. We have thus proved (i) $\Longleftrightarrow$ (iii). Since any $\lambda \in \Lambda$ can be chosen as a representative of its coset, the arguments above also show (i) $\Rightarrow$ (ii). Since (ii) $\Rightarrow$ (iii) is clear, the proof is complete.

To put Proposition 4.3 in perspective, we relate the graded vector spaces $\operatorname{gr}_{\alpha}(V)$ and $\operatorname{gr}_{\beta}(V)$ by means of a value-function-like map

$$
\alpha_{*}: \operatorname{gr}_{\beta}(V) \rightarrow \Gamma \cup\{\infty\}
$$

defined as follows: for $\xi \in \operatorname{gr}_{\beta}(V), \xi \neq 0$, let $\ell(\xi)$ be the homogeneous component of $\xi$ of least degree, and let $\lambda=\operatorname{deg}(\ell(\xi))$, so $\ell(\xi) \in V_{\lambda}^{\beta}$; then let

$$
\alpha_{*}(\xi)=\alpha_{\lambda}(\ell(\xi)) \in \lambda \subseteq \Gamma
$$

Let also $\alpha_{*}(0)=\infty$. For $x \in V$ we thus have

$$
\alpha_{*}\left(\widetilde{x}^{\beta}\right)=\alpha(x)
$$

where $\tilde{x}^{\beta}$ denotes the image of $x$ in $\operatorname{gr}_{\beta}(V)$.

A similar construction applies to the valuation $v$, and yields a map

$$
v_{*}: \operatorname{gr}_{w}(F) \rightarrow \Gamma \cup\{\infty\}
$$

which satisfies the same properties as a valuation, and such that the image $v_{*}(\rho)$ of any nonzero $\rho \in \operatorname{gr}_{w}(F)$ depends only on its homogeneous component of least degree. The map $\alpha_{*}$ deserves the name of a graded $v_{*}$-value function since it satisfies the following properties:

(i) $\alpha_{*}(\xi)=\infty$ if and only if $\xi=0$; if $\xi \neq 0$, then $\alpha_{*}(\xi)=\alpha_{*}(\ell(\xi))$ and $\varepsilon \circ \alpha_{*}(\xi)=\operatorname{deg} \ell(\xi)$

(ii) $\alpha_{*}(\xi+\eta) \geq \min \left(\alpha_{*}(\xi), \alpha_{*}(\eta)\right)$ for $\xi, \eta \in \operatorname{gr}_{\beta}(V)$;

(iii) $\alpha_{*}(\xi \rho)=\alpha_{*}(\xi)+v_{*}(\rho)$ for $\xi \in \operatorname{gr}_{\beta}(V)$ and $\rho \in \operatorname{gr}_{w}(F)$. 
We may thus consider the associated graded structure $\operatorname{gr}_{\alpha_{*}}\left(\operatorname{gr}_{\beta}(V)\right)$. If $x \in V$ satisfies $\beta(x)=\lambda$ and $\alpha(x)=\gamma$, we may identify

$$
\left(x+V_{>\lambda}^{\beta}\right)+\operatorname{gr}_{\beta}(V)_{>\gamma}^{\alpha_{*}}=x+V_{>\gamma}^{\alpha}
$$

thus

$$
\operatorname{gr}_{\alpha_{*}}\left(\operatorname{gr}_{\beta}(V)\right)=\operatorname{gr}_{\alpha}(V)
$$

We define $\alpha_{*}$ to be a graded $v_{*}$-norm if

$$
\left[\operatorname{gr}_{\alpha_{*}}\left(\operatorname{gr}_{\beta}(V)\right): \operatorname{gr}_{v_{*}}\left(\operatorname{gr}_{w}(F)\right)\right]=\left[\operatorname{gr}_{\beta}(V): \operatorname{gr}_{w}(F)\right]
$$

It is easy to check that this holds if and only if each $\alpha_{\lambda}$ is a $u$-norm. By an argument analogous to the one in [16, Prop. 2.5] for ungraded norms, one can check that if $\alpha_{*}$ is a graded norm, then for any graded subspace $\mathrm{W}$ of $\operatorname{gr}_{\beta}(V), \alpha_{*} \mid \mathrm{w}$ is a graded norm on W. Consequently, by dimension count, the functor $\mathrm{gr}_{\alpha_{*}}\left({ }_{-}\right)$preserves strict inclusions of graded subspaces of $\operatorname{gr}_{\beta}(V)$. Proposition 4.3 may be rephrased as follows: $\alpha$ is a $v$-norm if and only if $\beta$ is a $w$-norm and $\alpha_{*}$ is a graded $v_{*}$-norm. Indeed, if $\left(e_{i}\right)_{i=1}^{n}$ is a splitting base of $V$ for $\alpha$, then it is also a splitting base for $\beta$, and $\left(\widetilde{e}_{i}^{\beta}\right)_{i=1}^{n}$ is a splitting base of $\operatorname{gr}_{\beta}(V)$ for $\alpha_{*}$.

We now apply this construction to a finite-dimensional $F$-algebra $A$. If $\alpha: A \rightarrow$ $\Gamma \cup\{\infty\}$ is a surmultiplicative $v$-value function, then the coarser $w$-value function $\beta=\varepsilon \circ \alpha$ is clearly surmultiplicative, and the map $\alpha_{*}$ is also surmultiplicative, by an easy calculation using (4.6). The notions of gauge and tame gauge for graded norms are defined analogously to the ungraded cases.

Proposition 4.4 The map $\alpha$ is a v-gauge (resp. a tame $v$-gauge) if and only if $\beta$ is a $w$-gauge (resp. a tame $w$-gauge) and $\alpha_{*}$ is a graded $v_{*}$-gauge (resp. a tame graded $v_{*}$-gauge).

Proof Proposition 4.3 already shows that $\alpha$ is a $v$-norm if and only if $\beta$ is a $w$-norm and $\alpha_{*}$ is a graded $v_{*}$-norm. We noted above that $\alpha$ is surmultiplicative if and only if $\beta$ and $\alpha_{*}$ are surmultiplicative.

Suppose $\alpha$ is a $v$-gauge. Since $\operatorname{gr}_{\alpha_{*}}\left(\operatorname{gr}_{\beta}(A)\right)=\operatorname{gr}_{\alpha}(A)$ and $\operatorname{gr}_{\alpha}(A)$ is semisimple, it follows that $\operatorname{gr}_{\beta}(A)$ is semisimple. For, if $\mathrm{I}$ is a nontrivial nilpotent homogeneous left ideal of $\operatorname{gr}_{\beta}(A)$, then $\operatorname{gr}_{\alpha_{*}}(\mathrm{l})$ is a nontrivial nilpotent homogeneous left ideal of $\operatorname{gr}_{\alpha_{*}}\left(\operatorname{gr}_{\beta}(A)\right)$. Thus, $\beta$ is a $w$-gauge. Also, $\operatorname{gr}_{\alpha_{*}}\left(\operatorname{gr}_{\beta}(A)\right)$ is semisimple by hypothesis, hence $\alpha_{*}$ is a graded $v_{*}$-gauge. Conversely, if $\beta$ is a $w$-gauge and $\alpha_{*}$ is a graded $v_{*}$-gauge, then $\alpha$ is a $v$-gauge since $\operatorname{gr}_{\alpha}(A)=\operatorname{gr}_{\alpha_{*}}\left(\operatorname{gr}_{\beta}(A)\right)$.

Assume now that $\alpha$ is a $v$-gauge. For the centers we have the obvious inclusions

$$
\begin{aligned}
\operatorname{gr}_{\alpha}(Z(A)) & =\operatorname{gr}_{\alpha_{*}}\left(\operatorname{gr}_{\beta}(Z(A))\right) \subseteq \operatorname{gr}_{\alpha_{*}}\left(Z\left(\operatorname{gr}_{\beta}(A)\right)\right) \\
& \subseteq Z\left(\operatorname{gr}_{\alpha_{*}}\left(\operatorname{gr}_{\beta}(A)\right)\right)=Z\left(\operatorname{gr}_{\alpha}(A)\right)
\end{aligned}
$$


Thus, $Z\left(\operatorname{gr}_{\alpha}(A)\right)=\operatorname{gr}_{\alpha}(Z(A))$ if and only if we have equalities throughout (4.8); since $\operatorname{gr}_{\alpha_{*}}\left(\_\right)$preserves strict inclusions, this holds if and only if

$$
\operatorname{gr}_{\beta}(Z(A))=Z\left(\operatorname{gr}_{\beta}(A)\right) \text { and } \operatorname{gr}_{\alpha_{*}}\left(Z\left(\operatorname{gr}_{\beta}(A)\right)\right)=Z\left(\operatorname{gr}_{\alpha_{*}}\left(\operatorname{gr}_{\beta}(A)\right)\right)
$$

Assume we have these equalities. Let $Z=Z(A)$, which is a direct product of fields, as $A$ is semisimple. The separability condition on the graded center required for tameness holds for $\alpha$ if and only if it holds for $\alpha_{*}$, since they have the same graded rings. Suppose now that $\operatorname{gr}_{\beta}(Z)$ is not separable over $\operatorname{gr}_{w}(F)$. Because $\operatorname{gr}_{\beta}(A)$ is semisimple, its center $\operatorname{gr}_{\beta}(Z)$ is a direct product $\mathrm{C}_{1} \times \cdots \times \mathrm{C}_{k}$ of graded fields, and some $\mathrm{C}_{j}$ must not be separable over $\operatorname{gr}_{w}(F)$. By [10, Prop. 3.7, Prop. 3.5] there is a graded field T with $\mathrm{gr}_{w}(F) \subseteq \mathrm{T} \varsubsetneqq \mathrm{C}_{j}$ and $\mathrm{C}_{j}$ purely inseparable over $\mathrm{T}$. So,

$$
\operatorname{gr}_{v}(F)=\operatorname{gr}_{\alpha_{*}}\left(\operatorname{gr}_{w}(V)\right) \subseteq \operatorname{gr}_{\alpha_{*}}(\mathrm{~T}) \varsubsetneqq \operatorname{gr}_{\alpha_{*}}\left(\mathrm{C}_{j}\right)
$$

and $\operatorname{gr}_{\alpha_{*}}\left(\mathrm{C}_{j}\right)$ is purely inseparable over $\operatorname{gr}_{\alpha_{*}}(\mathrm{~T})$. Now,

$$
\operatorname{gr}_{\alpha}(Z)=\operatorname{gr}_{\alpha_{*}}\left(\operatorname{gr}_{\beta}(Z)\right)=\prod_{i=1}^{k} \operatorname{gr}_{\alpha_{*}}\left(\mathrm{C}_{i}\right)
$$

Since $\mathrm{gr}_{\alpha_{*}}\left(\mathrm{C}_{j}\right)$ is purely inseparable over $\mathrm{gr}_{\alpha_{*}}(\mathrm{~T})$, it cannot be separable over $\operatorname{gr}_{v}(F)$, so $\operatorname{gr}_{\alpha}(Z)$ is not separable over $\operatorname{gr}_{v}(F)$. Thus, $\operatorname{gr}_{\alpha}(Z)$ is separable over $\operatorname{gr}_{v}(F)$ if and only $\operatorname{gr}_{\alpha_{*}}\left(\operatorname{gr}_{\beta}(Z)\right)$ is separable over $\operatorname{gr}_{v_{*}}\left(\operatorname{gr}_{w}(F)\right)$ and $\operatorname{gr}_{\beta}(Z)$ is separable over $\operatorname{gr}_{w}(F)$. Therefore, $\alpha$ is a tame $v$-gauge if and only if $\beta$ is a tame $w$-gauge and $\alpha_{*}$ is a tame graded $v_{*}$-gauge.

\section{Descent of norms}

Throughout this section, we fix the following notation: $V$ is a finite-dimensional vector space over a field $F$, and $v: F \rightarrow \Gamma \cup\{\infty\}$ is a valuation. Let $\left(F_{h}, v_{h}\right)$ be a Henselization of $(F, v)$. If $\alpha: V \otimes_{F} F_{h} \rightarrow \Gamma \cup\{\infty\}$ is a $v_{h}$-norm, then clearly $\left.\alpha\right|_{V}: V \rightarrow \Gamma \cup\{\infty\}$ is a $v$-value function, but not necessarily a $v$-norm unless $\Gamma$ has rank one, see Proposition 5.4 and Example 5.6. In this section, we give an inductive criterion for $\left.\alpha\right|_{V}$ to be a $v$-norm when $\Gamma$ is the divisible hull of $\Gamma_{F}$ and the rank $\operatorname{rk}(\Gamma)$ is finite, see Proposition 5.5.

We first discuss the descent problem in a general context: let $\left(K, v_{K}\right)$ be an arbitrary valued field extension of $(F, v)$, and let $\alpha: V \otimes_{F} K \rightarrow \Gamma \cup\{\infty\}$ be a $v_{K}$-norm. We identify $V$ with its canonical image in $V \otimes_{F} K$. For any $x \in V$ and $c \in K$ we have

$$
\alpha(x \otimes c)=\alpha((x \otimes 1) \cdot c)=\left.\alpha\right|_{V}(x)+v_{K}(c)
$$

Therefore, for any $\gamma \in \Gamma_{V,\left.\alpha\right|_{V}}$ and $\delta \in \Gamma_{K}$ the usual $F$-bilinear map $V \times K \rightarrow V \otimes_{F} K$ sends $V_{\geq \gamma} \times K_{\geq \delta}$ into $\left(V \otimes_{F} K\right)_{\geq \gamma+\delta}$. Likewise, $V_{>\gamma} \times K_{\geq \delta}$ and $V_{\geq \gamma} \times K_{>\delta}$ map into $\left(V \otimes_{F} K\right)_{>\gamma+\delta}$. Consequently, there is a well- defined induced map $V_{\gamma} \times K_{\delta} \rightarrow$ $\left(V \otimes_{F} K\right)_{\gamma+\delta}$ given by $(\widetilde{x}, \widetilde{c}) \mapsto \widetilde{x \otimes c}$. The direct sum of these maps over all such 
$\gamma, \delta$ yields a homomorphism $\operatorname{gr}_{\left.\alpha\right|_{V}}(V) \times \operatorname{gr}_{v_{K}}(K) \rightarrow \operatorname{gr}_{\alpha}\left(V \otimes_{F} K\right)$, which is clearly $\operatorname{gr}_{v}(F)$-bilinear; hence there is a canonical map

$$
\chi: \operatorname{gr}_{\left.\alpha\right|_{V}}(V) \otimes \operatorname{gr}_{v}(F) \operatorname{gr}_{v_{K}}(K) \rightarrow \operatorname{gr}_{\alpha}\left(V \otimes_{F} K\right)
$$

which maps $\widetilde{x} \otimes \widetilde{c}$ to $\widetilde{x \otimes c}$ for $x \in V$ and $c \in K$.

On the other hand, recall from Sect. 1 (see (1.5)) that if $\left.\alpha\right|_{V}$ is a $v$-norm on $V$, then there is a canonical isomorphism of $\mathrm{gr}_{v_{K}}(K)$-vector spaces

$$
\rho: \operatorname{gr}_{\left.\alpha\right|_{V} \otimes v_{K}}\left(V \otimes_{F} K\right) \stackrel{\sim}{\rightarrow} \operatorname{gr}_{\left.\alpha\right|_{V}}(V) \otimes_{\operatorname{gr}_{v}(F)} \operatorname{gr}_{v_{K}}(K)
$$

which maps $\widetilde{x \otimes c}$ to $\tilde{x} \otimes \tilde{c}$ for $x \in V$ and $c \in K$.

Lemma 5.1 The following conditions are equivalent:

(a) $\left.\alpha\right|_{V}$ is a v-norm on $V$ and $\alpha=\left.\alpha\right|_{V} \otimes v_{K}$.

(b) $V$ contains a $K$-splitting base of the norm $\alpha$ on $V \otimes_{F} K$.

(c) $\left.\alpha\right|_{V}$ is a v-norm and the canonical map $\chi$ is injective.

When these conditions hold, the map $\chi$ is a graded isomorphism, which is the inverse of $\rho$, and $\Gamma_{V \otimes_{F} K, \alpha}=\Gamma_{V,\left.\alpha\right|_{V}}+\Gamma_{K, v_{K}}$.

Proof (b) $\Rightarrow$ (a) If $\mathcal{B}=\left(e_{i}\right)_{i=1}^{n} \subseteq V$ is a splitting base for $\alpha$ on $V \otimes_{F} K$, then $\mathcal{B}$ is clearly also a splitting base for $\left.\alpha\right|_{V}$ on $V$. So, $\left.\alpha\right|_{V}$ is a $v$-norm. Furthermore, by the definition of $\left.\alpha\right|_{V} \otimes v_{K}$, we have for any $k_{1}, \ldots, k_{n} \in K$,

$$
\begin{aligned}
\left(\left.\alpha\right|_{V} \otimes v_{K}\right)\left(\sum_{i=1}^{n} e_{i} \otimes k_{i}\right) & =\min _{1 \leq i \leq n}\left(\left.\alpha\right|_{V}\left(e_{i}\right)+v_{K}\left(k_{i}\right)\right) \\
& =\min _{1 \leq i \leq n}\left(\alpha\left(e_{i} \otimes 1\right)+v_{K}\left(k_{i}\right)\right) \\
& =\alpha\left(\sum_{i=1}^{n}\left(e_{i} \otimes 1\right) \cdot k_{i}\right)=\alpha\left(\sum_{i=1}^{n} e_{i} \otimes k_{i}\right),
\end{aligned}
$$

showing that $\left.\alpha\right|_{V} \otimes v_{K}=\alpha$.

(a) $\Rightarrow$ (c) When (a) holds, $\left.\alpha\right|_{V}$ is a norm, and $\chi$ is clearly the inverse of $\rho$, so $\chi$ is injective.

(c) $\Rightarrow$ (b) Suppose (c) holds. Let $\left(e_{i}\right)_{i=1}^{n}$ be an $F$-splitting base for $\left.\alpha\right|_{V}$ on $V$. Then, by [16, Cor. 2.3(ii)] $\widetilde{e_{1}}, \ldots, \widetilde{e_{n}}$ are $\operatorname{gr}_{v}(F)$-linearly independent in $\operatorname{gr}_{\left.\alpha\right|_{V}}(V)$. Hence,

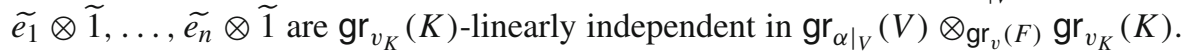

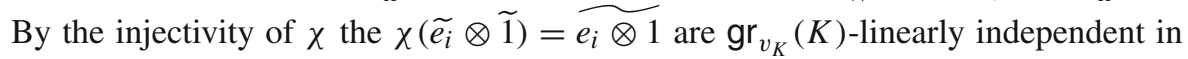
$\operatorname{gr}_{\alpha}\left(V \otimes_{F} K\right)$. But, since $\alpha$ and $\left.\alpha\right|_{V}$ are norms,

$$
\left[\operatorname{gr}_{\alpha}\left(V \otimes_{F} K\right): \operatorname{gr}_{v_{K}}(K)\right]=\left[V \otimes_{F} K: K\right]=[V: F]=n
$$

Therefore, $\left(\widetilde{e_{i} \otimes 1}\right)_{i=1}^{n}$ is a homogeneous $\operatorname{gr}_{v_{K}}(K)$-vector space base of $\operatorname{gr}_{\alpha}\left(V \otimes_{F} K\right)$, hence $\left(e_{i} \otimes 1\right)_{i=1}^{n}$ is a $K$-splitting base for $\alpha$ on $V \otimes_{F} K$ by [16, Cor. 2.3(ii)]. 
When the conditions (a) - (c) hold, we have

$$
\Gamma_{V \otimes_{F} K, \alpha}=\Gamma_{V \otimes_{F} K,\left.\alpha\right|_{V} \otimes v_{K}}=\Gamma_{V,\left.\alpha\right|_{V}}+\Gamma_{K, v_{K}}
$$

and the map $\chi$ is the inverse of $\rho$, so $\chi$ is an isomorphism.

Note that under the hypotheses of Lemma 5.1 if $\left.\alpha\right|_{V}$ is a norm then $\alpha \geq\left.\alpha\right|_{V} \otimes v_{K}$. For, if $\left(e_{i}\right)_{i=1}^{n}$ is a $v$-splitting base for $\left.\alpha\right|_{V}$ on $V$, then for any $k_{1}, \ldots, k_{n} \in K$,

$$
\begin{aligned}
\alpha\left(\sum_{i=1}^{n} e_{i} \otimes k_{i}\right) & \geq \min _{1 \leq i \leq n}\left(\alpha\left(e_{i} \otimes k_{i}\right)\right)=\min _{1 \leq i \leq n}\left(\alpha\left(e_{i}\right)+v_{K}\left(k_{i}\right)\right) \\
& =\left(\left.\alpha\right|_{V} \otimes v_{K}\right)\left(\sum_{i=1}^{n} e_{i} \otimes k_{i}\right) .
\end{aligned}
$$

We next show that the inequality $\alpha \geq\left.\alpha\right|_{V} \otimes v_{K}$ is actually an equality when $K$ is immediate over $F$, but not in general.

Corollary 5.2 Let $\left(K, v_{K}\right)$ be an immediate valued field extension of $(F, v)$ and let $\alpha: V \otimes_{F} K \rightarrow \Gamma \cup\{\infty\}$ be a $v_{K}$-norm. If $\left.\alpha\right|_{V}$ is a norm, then $\alpha=\left.\alpha\right|_{V} \otimes v_{K}$. So, the canonical map $\operatorname{gr}_{\left.\alpha\right|_{V}}(V) \rightarrow \operatorname{gr}_{\alpha}\left(V \otimes_{F} K\right)$ is an isomorphism $\operatorname{gr}_{\left.\alpha\right|_{V}}(V) \cong$ $\operatorname{gr}_{\alpha}\left(V \otimes_{F} K\right)$, and $\Gamma_{V}=\Gamma_{V \otimes_{F} K}$.

Proof Since $v_{K}$ is immediate over $v$, we have $\operatorname{gr}_{v_{K}}(K)=\operatorname{gr}_{v}(F)$, so the canonical map $\chi$ of Lemma 5.1(c) is just the injection $\mathrm{gr}_{\left.\alpha\right|_{V}}(V) \hookrightarrow \mathrm{gr}_{\alpha}\left(V \otimes_{F} K\right)$ arising from the canonical inclusion $V \hookrightarrow V \otimes_{F} K$. Thus, the corollary follows from Lemma 5.1, using $\Gamma_{K}=\Gamma_{F}$ for the last assertion.

Example 5.3 Let $\left(K, v_{K}\right)$ be an extension of $(F, v)$ with $\bar{F} \varsubsetneqq \bar{K}$. Let $\xi \in K$ be such that $v_{K}(\xi)=0$ and $\bar{\xi} \notin \bar{F}$, and let $V$ be a 2-dimensional $F$-vector space with base $\left(e_{1}, e_{2}\right)$. Let $f=e_{1} \otimes 1+e_{2} \otimes \xi \in V \otimes_{F} K$, and consider the $v_{K}$-norm $\alpha$ on $V \otimes_{F} K$ with splitting base $\left(e_{1} \otimes 1, f\right)$ such that

$$
\alpha\left(e_{1} \otimes 1\right)=0 \quad \text { and } \quad \alpha(f)>0
$$

Then, as $e_{2}=\left(f-e_{1}\right) \xi^{-1}$, we have for $c_{1}, c_{2} \in F$,

$$
\begin{aligned}
\left.\alpha\right|_{V}\left(e_{1} c_{1}+e_{2} c_{2}\right) & =\alpha\left(\left(e_{1}\left(c_{1}-\xi^{-1} c_{2}\right)+f c_{2}\right)\right. \\
& =\min \left(\alpha\left(e_{1}\right)+v_{K}\left(c_{1}-\xi^{-1} c_{2}\right), \alpha(f)+v_{K}\left(c_{2}\right)\right) \\
& =\min \left(v\left(c_{1}\right), v\left(c_{2}\right)\right) .
\end{aligned}
$$

Hence, $\left(e_{1}, e_{2}\right)$ is a $v$-splitting base of $V$ for $\left.\alpha\right|_{V}$, showing that $\left.\alpha\right|_{V}$ is a $v$-norm on $V$. However, $\left.\alpha\right|_{V} \otimes v_{K}<\alpha$ since

$$
\left(\left.\alpha\right|_{V} \otimes v_{K}\right)(f)=\min \left(v_{K}(1), v_{K}(\xi)\right)=0<\alpha(f) .
$$


Thus, the first condition in Lemma 5.1(a) holds, but not the second. The Lemma shows that $V$ does not contain any splitting base for the $v_{K}$-norm $\alpha$ on $V \otimes_{F} K$. Also, the second condition in part (c) of the Lemma fails, since the canonical map $\chi$ satisfies

$$
\chi\left(\widetilde{e_{1}} \otimes 1+\widetilde{e_{2}} \otimes \widetilde{\xi}\right)=\widetilde{e_{1} \otimes 1}+\widetilde{e_{2} \otimes \xi}=0 .
$$

We now turn to the descent problem posed at the beginning of this section, for $\left(K, v_{K}\right)=\left(F_{h}, v_{h}\right)$ a Henselization of $(F, v)$. The rank one case is easy:

Proposition 5.4 Let $\alpha: V \otimes_{F} F_{h} \rightarrow \Gamma \cup\{\infty\}$ be a $v_{h}$-norm, let $\gamma \in \Gamma$, and suppose $\operatorname{im}(\alpha) \subseteq \gamma+\left(\Gamma_{F} \otimes_{\mathbb{Z}} \mathbb{Q}\right)$. If $\operatorname{rk}\left(\Gamma_{F}\right)=1$, then $\left.\alpha\right|_{V}$ is a $v$-norm and $\alpha=\left.\alpha\right|_{V} \otimes v_{h}$.

Proof Let $\left(e_{i}\right)_{i=1}^{n}$ be an arbitrary $F$-base of $V$ and let $x \in V \otimes_{F} F_{h}$,

$$
x=\sum_{i=1}^{n} e_{i} \otimes k_{i} \quad \text { for some } k_{i} \in F_{h} .
$$

Since $\operatorname{rk}\left(\Gamma_{F}\right)=1$, the field $F$ is dense in $F_{h}$ for the topology of the valuation $v_{h}$ : see [7, Sect. 1.6] or use the fact that $F$ is dense in its completion $\widehat{F}$ and that $F_{h}$ embeds in $\widehat{F}$ by [8, Th. 17.18]. Furthermore, $\Gamma_{F}$ is dense in its divisible hull $\Gamma_{F} \otimes_{\mathbb{Z}} \mathbb{Q}$. For each $i, 1 \leq i \leq n$, since $\alpha(x)-\alpha\left(e_{i} \otimes 1\right) \in \Gamma_{F} \otimes_{\mathbb{Z}} \mathbb{Q}$, we may therefore find an element $f_{i} \in F$ such that

$$
v_{h}\left(k_{i}-f_{i}\right)>\alpha(x)-\alpha\left(e_{i} \otimes 1\right) .
$$

Let $y=\sum_{i=1}^{n} e_{i} \otimes f_{i}=\sum_{i=1}^{n} e_{i} f_{i} \otimes 1 \in V$. Then,

$$
\begin{aligned}
\alpha(x-y) & =\alpha\left(\sum_{i=1}^{n} e_{i} \otimes\left(k_{i}-f_{i}\right)\right) \geq \min _{1 \leq i \leq n}\left(\alpha\left(e_{i} \otimes\left(k_{i}-f_{i}\right)\right)\right) \\
& =\min _{1 \leq i \leq n}\left(\alpha\left(e_{i}\right)+v_{h}\left(k_{i}-f_{i}\right)\right)>\alpha(x) .
\end{aligned}
$$

Hence,

$$
\tilde{x}=\widetilde{y} \in \operatorname{gr}_{\left.\alpha\right|_{V}}(V) \text {. }
$$

This proves that the monomorphism $\mathrm{gr}_{\left.\alpha\right|_{V}}(V) \hookrightarrow \operatorname{gr}_{\alpha}\left(V \otimes_{F} F_{h}\right)$ is an isomorphism. Hence, as $\alpha$ is a norm,

$$
\begin{aligned}
\left.\operatorname{cgr}_{\left.\alpha\right|_{V}}(V): \operatorname{gr}_{v}(F)\right] & =\left[\operatorname{gr}_{\alpha}\left(V \otimes_{F} F_{h}\right): \operatorname{gr}_{v_{h}}\left(F_{h}\right)\right] \\
& =\left[\left(V \otimes_{F} F_{h}\right): F_{h}\right]=[V: F],
\end{aligned}
$$

which shows that $\left.\alpha\right|_{V}$ is a $v$-norm. 
Now, suppose $\Gamma=\Gamma_{F} \otimes_{\mathbb{Z}} \mathbb{Q}$, with $\operatorname{rk}(\Gamma)>1$, and suppose $\Gamma$ contains a convex subgroup $\Delta$ of rank 1 . As in Sect. 4 , we consider the canonical map $\varepsilon: \Gamma \rightarrow \Gamma / \Delta=\Lambda$ and the coarser valuation

$$
w=\varepsilon \circ v: F \rightarrow \Lambda \cup\{\infty\}
$$

Let $\left(F_{h, v}, v_{h}\right)$ be a Henselization of $(F, v)$ and $\left(F_{h, w}, w_{h}\right)$ a Henselization of $(F, w)$. Let also

$$
y=\varepsilon \circ v_{h}: F_{h, v} \rightarrow \Lambda \cup\{\infty\} .
$$

By [9, Cor. 4.1.4, p. 90], the valuation $y$ is Henselian, hence we may assume $\left(F_{h, w}, w_{h}\right) \subseteq\left(F_{h, v}, y\right)$.

Let $\alpha: V \otimes_{F} F_{h, v} \rightarrow \Gamma \cup\{\infty\}$ be a $v_{h}$-norm, and let

$$
\beta=\varepsilon \circ \alpha: V \otimes_{F} F_{h, v} \rightarrow \Lambda \cup\{\infty\}
$$

By Proposition 4.3, the map $\beta$ is a $y$-norm.

Proposition 5.5 If $\left.\beta\right|_{V}$ is a w-norm and $\beta=\left.\beta\right|_{V} \otimes y$, then $\left.\alpha\right|_{V}$ is a $v$-norm on $V$ and $\alpha=\left.\alpha\right|_{V} \otimes v_{h}$.

Proof As observed in Sect. 4, the valuation $v$ induces a valuation $u$ on the residue field $\bar{F}^{w}$,

$$
u: \bar{F}^{w} \rightarrow \Delta \cup\{\infty\}
$$

Note that the value group of $u$ is $\Delta_{F}=\Gamma_{F} \cap \Delta$ and, as $\Delta$ is divisible and torsion-free,

$$
\Delta_{F} \otimes_{\mathbb{Z}} \mathbb{Q}=\left(\Gamma_{F} \otimes_{\mathbb{Z}} \mathbb{Q}\right) \cap\left(\Delta \otimes_{\mathbb{Z}} \mathbb{Q}\right)=\Gamma \cap \Delta=\Delta .
$$

Let $\Lambda_{V}=\left.\beta\right|_{V}(V \backslash\{0\}) \subseteq \Lambda$. Clearly, $\left.\beta\right|_{V}=\varepsilon \circ\left(\left.\alpha\right|_{V}\right)$. In order to show that $\left.\alpha\right|_{V}$ is a norm, it therefore suffices, by Proposition 4.3 , to show that each map

$$
\left(\left.\alpha\right|_{V}\right)_{\lambda}: V_{\lambda}^{\left.\beta\right|_{V}} \rightarrow \lambda \cup\{\infty\}, \quad \text { for } \lambda \in \Lambda_{V},
$$

is a $u$-norm. To simplify notation, we write $V_{\lambda}$ for $V_{\lambda}^{\left.\beta\right|_{V}}$. Note that the canonical inclusion $V \hookrightarrow V \otimes_{F} F_{h, v}$ is compatible with the respective value functions $\left.\beta\right|_{V}$ and $\beta$ so yields an injection $V_{\lambda} \hookrightarrow\left(V \otimes_{F} F_{h, v}\right)_{\lambda}^{\beta}$; let $V_{\lambda}^{\prime}$ denote the image of $V_{\lambda}$. Then, clearly $\left.\alpha_{\lambda}\right|_{V_{\lambda}^{\prime}} \cong\left(\left.\alpha\right|_{V}\right)_{\lambda}$

Let $u_{h}:{\overline{F_{h, v}}}^{y} \rightarrow \Delta \cup\{\infty\}$ be the valuation induced by $v_{h}$. As observed by Morandi [14, p. 239], $\left(\bar{F}_{h, v} y, u_{h}\right)$ is a Henselization of $\left(\bar{F}^{w}, u\right)$. Since $\alpha$ is a $v_{h}$-norm, Proposition 4.3 shows that

$$
\alpha_{\lambda}:\left(V \otimes_{F} F_{h, v}\right)_{\lambda}^{\beta} \rightarrow \lambda \cup\{\infty\}
$$


is a $u_{h}$-norm for every $\lambda \in \Lambda_{V}$. Since $\left(F_{h, v}, y\right)$ is an inertial extension of $\left(F_{h, w}, w_{h}\right)$ by $[14$, p. 239], we have

$$
\begin{aligned}
\operatorname{gr}_{y}\left(F_{h, v}\right) & \cong \operatorname{gr}_{w_{h}}\left(F_{h, w}\right) \otimes \operatorname{gr}_{w_{h}}\left(F_{h, w}\right)_{0} \operatorname{gr}_{y}\left(F_{h, v}\right)_{0} \\
& =\operatorname{gr}_{w}(F) \otimes \operatorname{gr}_{w}(F)_{0} \operatorname{gr}_{y}\left(F_{h, v}\right)_{0} .
\end{aligned}
$$

Because $\beta=\left.\beta\right|_{V} \otimes y$, this yields graded isomorphisms

$$
\begin{aligned}
\operatorname{gr}_{\beta}\left(V \otimes_{F} F_{h, v}\right) & \cong \operatorname{gr}_{\left.\beta\right|_{V}}(V) \otimes_{\operatorname{gr}_{w}(F)} \operatorname{gr}_{y}\left(F_{h, v}\right) \\
& \cong \operatorname{gr}_{\left.\beta\right|_{V}}(V) \otimes_{\operatorname{gr}_{w}(F)_{0}} \operatorname{gr}_{y}\left(F_{h, v}\right)_{0} \cong \operatorname{gr}_{\left.\beta\right|_{V}}(V) \otimes_{\bar{F}^{w}}{\overline{F_{h, v}}}^{y} .
\end{aligned}
$$

For any $\lambda \in \Lambda_{F}$, when we restrict these graded isomorphisms to the $\lambda$-component we obtain the ${\overline{F_{h, v}}}$-vector space isomorphism

$$
\psi:\left(V \otimes_{F} F_{h, v}\right)_{\lambda}^{\beta} \stackrel{\sim}{\rightarrow} V_{\lambda} \otimes_{\bar{F}}^{w}{\overline{F_{h, v}}}^{y} .
$$

Let $\widehat{\alpha}=\alpha_{\lambda} \circ \psi^{-1}: V_{\lambda} \otimes_{\bar{F}}^{w}{\overline{F_{h, v}}}^{y} \rightarrow \lambda \cup\{\infty\}$, which is the $u_{h}$-value function on $\operatorname{im}(\psi)$ corresponding to $\alpha_{\lambda}$ on the domain of $\psi$. Since $\alpha_{\lambda}$ is a $u_{h}$-norm, so is $\widehat{\alpha}$. Because $\left({\overline{F_{h, v}}}^{y}, u_{h}\right)$ is a Henselization of $\left(\bar{F}^{w}, u\right)$ and $\lambda$ is a coset of $\Delta=\Delta_{F} \otimes_{\mathbb{Z}} \mathbb{Q}$, which has rank 1 , with $\Delta_{F}$ the value group of $u$, Proposition 5.4 applies to $\widehat{\alpha}$, and shows that $\left.\widehat{\alpha}\right|_{V_{\lambda}}$ is a $u$-norm. Note that $\psi$ maps the $V_{\lambda}^{\prime}$ defined above after (5.1) to the copy of $V_{\lambda}$ in $\operatorname{im}(\psi)$. So, $\left.\left.\alpha_{\lambda}\right|_{V_{\lambda}^{\prime}} \cong \widehat{\alpha}\right|_{V_{\lambda}}$. But, we saw above that $\left.\left(\left.\alpha\right|_{V}\right)_{\lambda} \cong \alpha_{\lambda}\right|_{V_{\lambda}^{\prime}}$. Since $\left.\widehat{\alpha}\right|_{V_{\lambda}}$ is a $u$-norm, these isomorphisms show that $\left(\left.\alpha\right|_{V}\right)_{\lambda}$ is also a $u$-norm. Thus, by Proposition $\left.4.3 \alpha\right|_{V}$ is a $v$-norm; then $\alpha=\left.\alpha\right|_{V} \otimes v_{h}$ by Corollary 5.2.

The following is an example of a norm on a Henselization that does not descend to a norm.

Example 5.6 Let $k$ be any field with $\operatorname{char}(k) \neq 2$, and let $F=k(x, y)$ with $x$ and $y$ algebraically independent over $k$. Let $v$ be the valuation on $F$ obtained by restriction from the canonical Henselian valuation on $k((x))((y))$, so $\Gamma_{F}=\mathbb{Z} \times \mathbb{Z}$ and $\bar{F}=k$. Let $\left(F_{h}, v_{h}\right)$ be a Henselization of $(F, v)$. Let $A=\left(\frac{1+x, y}{F}\right)$, a quaternion division algebra over $F$, and let $A_{h}=A \otimes_{F} F_{h}$. The algebra $A_{h}$ is split since $1+x \in F_{h}^{\times 2}$. Therefore, we may find $v_{h}$-gauges on $A_{h}$ that are unramified, in the sense that $\Gamma_{A_{h}}=\Gamma_{F}$. Fix such a $v_{h}$-gauge $\alpha$. We claim that $\left.\alpha\right|_{A}$ is not a $v$-norm on $A$.

Suppose the contrary. Then $\operatorname{gr}_{\left.\alpha\right|_{A}}(A)=\operatorname{gr}_{\alpha}\left(A_{h}\right)$ by Lemma 5.2, so $\left.\alpha\right|_{A}$ is a $v$-gauge. Consider the convex subgroup $\Delta=\mathbb{Z} \times\{0\} \subseteq \Gamma_{F}$ and the canonical epimorphism

$$
\varepsilon: \Gamma_{F} \rightarrow \Gamma_{F} / \Delta=\mathbb{Z}
$$

Let $w=\varepsilon \circ v: F \rightarrow \mathbb{Z} \cup\{\infty\}$, which is the $y$-adic valuation on $F$, and let $\beta=\varepsilon \circ \alpha: A_{h} \rightarrow \mathbb{Z} \cup\{\infty\}$. Proposition 4.4 shows that $\left.\beta\right|_{A}$ is a tame $w$-gauge on $A$. However, the $y$-adic valuation $w$ extends to $A$, so by [18, Cor. 3.4] $\left.\beta\right|_{A}$ is the 
(unique) valuation on $A$ that extends $w$. In particular, if $j \in A$ satisfies $j^{2}=y$ we must have $\left.\beta\right|_{A}(j)=\frac{1}{2}$. This is a contradiction since $\left.\beta\right|_{A}$ is unramified.

\section{Non-Henselian valuations}

Let $(F, v)$ be a valued field and let $A$ be a finite-dimensional simple $F$-algebra with an involution $\sigma$. Let $K=Z(A)$, and assume $F$ is the subfield of $K$ fixed under $\sigma$. Fix a Henselization $\left(F_{h}, v_{h}\right)$ of $(F, v)$.

Theorem 6.1 Suppose $A$ is split by the maximal tamely ramified extension of $F_{h}$. Moreover, if $\operatorname{char}(\bar{F})=2$ suppose that $\sigma$ is not an orthogonal involution. Then, the following conditions are equivalent:

(a) $\sigma \otimes \mathrm{id}_{F_{h}}$ is an anisotropic involution on $A \otimes_{F} F_{h}$;

(b) there exists a $\sigma$-special v-gauge $\varphi$ on $A$ i.e., $\varphi(\sigma(x) x)=2 \varphi(x)$ for all $x \in A$.

When they hold, $\varphi$ is the unique $v$-gauge on A that is invariant under $\sigma$, it is tame, and its value group lies in the divisible hull of $\Gamma_{F}$.

Proof Let $A_{h}=A \otimes_{F} F_{h}$ and $\sigma_{h}=\sigma \otimes \operatorname{id}_{F_{h}}$. If $\varphi$ is a $\sigma$-special $v$-gauge on $A$, then by Proposition $1.1 \varphi$ is invariant under $\sigma$ and $\widetilde{\sigma}$ is anisotropic on $\operatorname{gr}_{\varphi}(A)$. By Corollary $1.4, \varphi \otimes v_{h}$ is invariant under $\sigma_{h}$. Since

$$
\operatorname{gr}_{\varphi \otimes v_{h}}\left(A_{h}\right) \cong \operatorname{gr}_{\varphi}(A) \otimes \operatorname{gr}_{v}(F) \operatorname{gr}_{v_{h}}\left(F_{h}\right) \cong \operatorname{gr}_{\varphi}(A)
$$

and $\widetilde{\sigma_{h}} \cong \widetilde{\sigma}$, it follows that $\widetilde{\sigma_{h}}$ is anisotropic, hence $\sigma_{h}$ must also be anisotropic, proving (b) $\Rightarrow$ (a).

Now, suppose (a) holds. Let $\varphi_{1}$ and $\varphi_{2}$ be $v$-gauges on $A$ that are each invariant under $\sigma$. Then, by Corollary 1.4 each $\varphi_{i} \otimes v_{h}$ is a surmultiplicative $v_{h}$-norm on $A_{h}$ which is invariant under $\sigma_{h}$. Moreover, $\varphi_{i} \otimes v_{h}$ is a gauge on $A_{h}$ since $\operatorname{gr}_{\varphi_{i} \otimes v_{h}}\left(A_{h}\right) \cong$ $\operatorname{gr}_{\varphi_{i}}(A) \otimes \operatorname{gr}_{v}(F) \operatorname{gr}_{v_{h}}\left(F_{h}\right) \cong \operatorname{gr}_{\varphi_{i}}(A)$ and $\varphi_{i}$ is a gauge on $A$. Since $\sigma_{h}$ is assumed anisotropic, the uniqueness part of Theorem 2.2 (applied to $\sigma_{h}$ on $A_{h}$ ) yields $\varphi_{1} \otimes v_{h}=$ $\varphi_{2} \otimes v_{h}$, hence $\varphi_{1}=\varphi_{2}$. Theorem 2.2 also shows that $\varphi_{1} \otimes v_{h}$ is tame and satisfies $\varphi_{1}\left(\sigma_{h}(x) x\right)=2 \varphi_{1}(x)$ for all $x \in A_{h}$, hence $\varphi_{1}$ is tame and satisfies condition (b). Furthermore, $\Gamma_{A, \varphi_{1}}=\Gamma_{A_{h}, \varphi_{1} \otimes v_{h}}$ which lies in the divisible hull of $\Gamma_{F_{h}}=\Gamma_{F}$ by Theorem 2.2.

Thus, it only remains to prove the existence of a $v$-gauge on $A$ invariant under $\sigma$, assuming $\sigma_{h}$ is anisotropic. Note first that $K \otimes_{F} F_{h}$ is a field. For, otherwise, as $K$ is Galois over $F$ with $[K: F]=2, K \otimes_{F} F_{h}$ would be a direct sum of two fields, and the nontrivial $F_{h}$-automorphism $\left.\sigma\right|_{K \otimes_{F} F_{h}}$ must permute the two primitive idempotents of $K \otimes_{F} F_{h}$, call them $e_{1}$ and $e_{2}$. Then, $\sigma_{h}\left(e_{1}\right) e_{1}=e_{2} e_{1}=0$; but, this cannot happen as $\sigma_{h}$ is anisotropic. Since $K \otimes_{F} F_{h}$ is a field and $K=Z(A), A_{h} \cong A \otimes_{K}\left(K \otimes_{F} F_{h}\right)$ is a central simple $K \otimes_{F} F_{h}$-algebra.

Because $A_{h}$ is simple, $\sigma_{h}$ is anisotropic, and $v_{h}$ is Henselian, Theorem 2.2 yields a $\sigma_{h}$-invariant $v_{h}$-gauge $\varphi_{h}$ on $A_{h}$ whose value set lies in the divisible hull of $\Gamma_{F_{h}}=\Gamma_{F}$. The restriction $\varphi=\left.\varphi_{h}\right|_{A}$ is clearly a $\sigma$-invariant $v$-value function whose value set lies in the divisible hull of $\Gamma_{F}$. Henceforth, we may thus assume $\Gamma=\Gamma_{F} \otimes_{\mathbb{Z}} \mathbb{Q}$. If we show 
that $\varphi$ is a $v$-norm, then Corollary 5.2 yields $\varphi_{h}=\varphi \otimes v_{h}$, $\operatorname{sogr}_{\varphi}(A)=\operatorname{gr}_{\varphi \otimes v_{h}}\left(A_{h}\right)=$ $\operatorname{gr}_{\varphi_{h}}\left(A_{h}\right)$, hence $\varphi$ is a $v$-gauge, and the proof will be complete.

Suppose first that $\operatorname{rk}\left(\Gamma_{F}\right)<\infty$. We then argue by induction on $\operatorname{rk}\left(\Gamma_{F}\right)$. If $\operatorname{rk}\left(\Gamma_{F}\right)=$ 1 , then Proposition 5.4 shows that $\varphi$ is a $v$-norm. So, we may assume $\operatorname{rk}\left(\Gamma_{F}\right)>1$. Let $\Delta \subseteq \Gamma$ be a convex subgroup of rank 1 and let

$$
\varepsilon: \Gamma \rightarrow \Lambda=\Gamma / \Delta
$$

be the canonical epimorphism. Let $w=\varepsilon \circ v$ and $y=\varepsilon \circ v_{h}$, to agree with the notation of Sect. 5. So, $w$ has value group $\Lambda_{F}=\left(\Gamma_{F}+\Delta\right) / \Delta$, and $\Lambda=\Lambda_{F} \otimes_{\mathbb{Z}} \mathbb{Q}$, which has rank $\operatorname{rk}\left(\Gamma_{F}\right)-1$. Let $\left(F_{h, w}, w_{h}\right) \subseteq\left(F_{h}, y\right)$ be a Henselization of $(F, w)$. Since $\sigma_{h}$ is anisotropic, its restriction $\sigma \otimes \operatorname{id}_{F_{h, w}}$ is an anisotropic involution on the subring $A \otimes_{F} F_{h, w}$ of $A_{h}$. Since $A_{h} \cong\left(A \otimes_{F} F_{h, w}\right) \otimes_{F_{h, w}} F_{h}$ and $A_{h}$ is simple, $A \otimes_{F} F_{h, w}$ must also be simple. Therefore, Theorem 2.2 applies, yielding a $w_{h}$-gauge $\psi_{h}$ on $A \otimes_{F} F_{h, w}$ invariant under $\sigma \otimes \mathrm{id}_{F_{h, w}}$. By induction, $\left.\psi_{h}\right|_{A}$ is a $w$-gauge on $A$ invariant under $\sigma$. The same argument as for $\varphi_{1}$ above shows that the gauge $\left.\psi\right|_{A}$ is tame. Therefore, by [18, Cor. 1.26] $\left.\psi_{h}\right|_{A} \otimes y$ is a $y$-gauge on $A_{h}$, which is $\sigma_{h}$-invariant by Corollary 1.4. But, $\varepsilon \circ \varphi_{h}$ is also a $y$-gauge on $A_{h}$, by Proposition 4.4 since $\varphi$ is a gauge, and $\varepsilon \circ \varphi_{h}$ is invariant under $\sigma_{h}$ because $\varphi_{h}$ is. By the uniqueness given in Theorem 2.2, it follows that $\varepsilon \circ \varphi_{h}=\left.\psi_{h}\right|_{A} \otimes y$. Restricting to $A$, we also have $\varepsilon \circ \varphi=\left.\psi_{h}\right|_{A}$, which is a $w$-gauge so a $w$-norm on $A$. Furthermore, $(\varepsilon \circ \varphi) \otimes y=\left.\psi_{h}\right|_{A} \otimes y=\varepsilon \circ \varphi_{h}$. Proposition 5.5 with $\alpha=\varphi_{h}$ then shows that $\varphi$ is a $v$-norm. The theorem is thus proved if $\operatorname{rk}\left(\Gamma_{F}\right)<\infty$.

For the rest of the proof, assume that $\Gamma_{F}$ has infinite rank. Let $\left(a_{i}\right)_{i=1}^{n}$ be an $F$-base of $A$. Write $a_{i} a_{k}=\sum_{l} c_{i k l} a_{l}$ for some $c_{i k l} \in F$ and $\sigma\left(a_{i}\right)=\sum_{k} d_{i k} a_{k}$ for some $d_{i k} \in F$. Let $F_{0}$ be the prime subfield of $F$, and let

$$
F_{1}=F_{0}\left(\left\{c_{i k l}, d_{i k} \mid 1 \leq i, k, l \leq n\right\}\right) \subseteq F .
$$

Let $A_{1}$ be the $F_{1}$-span of the $a_{i}$, which is an $F_{1}$-algebra. We have $A_{1} \otimes_{F_{1}} F=A$ and $\sigma$ restricts to an involution $\sigma_{1}$ on $A_{1}$. Now, let $\left(e_{i}\right)_{i=1}^{n}$ be a splitting base of $A_{h}$ for the $v$-norm $\varphi_{h}$. We need to enlarge $F_{1}$ to capture the $e_{i}$ in the Henselization: let $L$ be any field with $F_{1} \subseteq L \subseteq F$ and $L$ finitely generated over $F_{1}$, and let $v_{L}=\left.v\right|_{L}$. Since $F_{h}$ is Henselian, there is a unique Henselization $\left(L_{h}, v_{L, h}\right)$ of $\left(L, v_{L}\right)$ inside $\left(F_{h}, v_{h}\right)$ by [9, Th. 5.2.2(2), p. 121]. Because $F$ is the direct limit of such fields $L$, the direct limit over such $L$ of the $\left(L_{h}, v_{L, h}\right)$ is a Henselian valued field $\left(M, v_{M}\right)$ with $F \subseteq M \subseteq F_{h}$ and $\left.v_{h}\right|_{M}=v_{M}$. Therefore, $\left(M, v_{M}\right)=\left(F_{h}, v_{h}\right)$ by the uniqueness of the Henselization. Since $A_{h}=A_{1} \otimes_{F_{1}} M$, there is a field $F_{2}$ finitely generated over $F_{1}$ (hence also over $\left.F_{0}\right)$ such that $e_{1}, \ldots, e_{n} \in A_{1} \otimes_{F_{1}}\left(F_{2}\right)_{h}$. Let

$$
A_{2}=A_{1} \otimes_{F_{1}} F_{2} \subseteq A, \quad A_{2, h}=A_{1} \otimes_{F_{1}}\left(F_{2}\right)_{h}=A_{2} \otimes_{F_{2}}\left(F_{2}\right)_{h} \subseteq A_{h} .
$$

Note that $A_{2}$ is a simple $F_{2}$-algebra since $A=A_{F_{2}} \otimes_{F_{2}} F$ and $A$ is simple. Let $\sigma_{2}=\left.\sigma\right|_{A_{2}}$, which is an involution on $A_{2}$, and let

$$
\sigma_{2, h}=\left.\sigma_{h}\right|_{A_{2, h}}=\sigma_{2} \otimes \operatorname{id}_{\left(F_{2}\right)_{h}},
$$


which is an anisotropic involution on $A_{2, h}$. Let $\varphi_{2}=\left.\varphi_{h}\right|_{A_{2}}$ and $\varphi_{2, h}=\left.\varphi_{h}\right|_{A_{2, h}}$. Since $A_{h}=A_{2, h} \otimes_{\left(F_{2}\right)_{h}} F_{h}$ and $e_{1}, \ldots, e_{n} \in A_{2, h}$, Lemma 5.1 says that $\varphi_{2, h}$ is a $v_{F_{2}, h}$-norm on $A_{2, h}$ and $\varphi_{h}=\varphi_{2, h} \otimes v_{h}$. Now, $F_{2}$ is finitely generated over the prime field $F_{0}$, so $\operatorname{rk}\left(\Gamma_{F_{2}, v_{F_{2}}}\right) \leq \operatorname{trdeg}\left(F_{2} / F_{0}\right)<\infty$ by [1, Ch. 6, Sect. 10.3, Cor. 2]. Since $\varphi_{2, h}$ is a $v_{F_{2}, h}$-norm, the finite rank case shows that $\varphi_{2}$ is a $v_{F_{2}}$-norm on $A_{2}$; then, $\varphi_{2, h}=\varphi_{2} \otimes v_{F_{2}, h}$ by Corollary 5.2. Hence,

$$
\varphi_{2} \otimes v_{h}=\left(\varphi_{2} \otimes v_{F_{2}, h}\right) \otimes v_{h}=\varphi_{2, h} \otimes v_{h}=\varphi_{h} .
$$

Therefore, as $\varphi_{2}$ is a norm, $\left.\varphi_{h}\right|_{A}=\left.\left(\varphi_{2} \otimes v_{h}\right)\right|_{A_{2} \otimes_{F_{2}} F}=\varphi_{2} \otimes v$, which is a norm since it is a scalar extension of the norm $\varphi_{2}$.

Corollary 6.2 With the hypotheses on A, $\sigma$, and $v$ as in Theorem 6.1, let $\varphi$ be a $v$-gauge on A which is invariant under $\sigma$. Then,

(a) If the residue involution $\sigma_{0}$ is anisotropic, then $\varphi$ is the unique $\sigma$-special $v$-gauge on $A$.

(b) If $\sigma_{0}$ is isotropic, then there is no $\sigma$-special v-gauge on A.

Proof Let $\left(F_{h}, v_{h}\right)$ be a Henselization of $(F, v)$, and let $A_{h}=A \otimes_{F} F_{h}$ and $\sigma_{h}=$ $\sigma \otimes \operatorname{id}_{F_{h}}$. Let $\varphi_{h}=\varphi \otimes v_{h}$, a surmultiplicative value function on $A_{h}$ which is invariant under the involution $\sigma_{h}$, by Corollary 1.4. The graded isomorphisms $\operatorname{gr}_{\varphi_{h}}\left(A_{h}\right) \cong$ $\operatorname{gr}_{\varphi}(A) \otimes \operatorname{gr}_{v}(F) \operatorname{gr}_{v_{h}}\left(F_{h}\right) \cong \operatorname{gr}_{\varphi}(A)$ show that $\varphi_{h}$ is a gauge on $A_{h}$, and $\widetilde{\sigma}_{h} \cong \widetilde{\sigma}$ and $\left(\sigma_{h}\right)_{0} \cong \sigma_{0}$. (a) If $\sigma_{0}$ is anisotropic, then so is $\left(\sigma_{h}\right)_{0}$, and so also is $\sigma_{h}$ by Corollary 2.3. Theorem 6.1 then shows that $\varphi$ is a $\sigma$-special $v$-gauge and is the unique such $v$-gauge on $A$, proving (a). For (b), we prove the contrapositive: If there were a $\sigma$-special $v$-gauge $\psi$ for $A$ then the uniqueness in Theorem 6.1 shows that $\varphi=\psi$. Hence, $\sigma_{h}$ is anisotropic by Theorem 6.1, so $\left(\sigma_{h}\right)_{0}$ is anisotropic by Corollary 2.3, which implies $\sigma_{0}$ is anisotropic as well.

Example 6.3 Even when there is no $\sigma$-special $v$-gauge on $A$, there may still be tame $v$-gauges on $A$ invariant under $\sigma$, but they need not be unique. For example, let $A$ the quaternion division algebra $(-1,-1) \mathbb{Q}$ over the field of rational numbers, and let $v$ be the 3 -adic valuation on $\mathbb{Q}$. Let $(1, i, j, k)$ be the quaternion base of $A$ with $i^{2}=j^{2}=-1$ and $k=i j=-j i$. As shown in [18, Ex. 1.16], a $v$-gauge $\varphi$ can be defined on $A$ by

$$
\varphi\left(a_{0}+a_{1} i+a_{2} j+a_{3} k\right)=\min \left(v\left(a_{0}\right), v\left(a_{1}\right), v\left(a_{2}\right), v\left(a_{3}\right)\right) .
$$

Clearly, the residue algebra of $A$ for $\varphi$ is $A_{0}=(-1,-1)_{\mathbb{F}_{3}} \cong M_{2}\left(\mathbb{F}_{3}\right)$. The $v$-gauge $\varphi$ is obviously invariant under the conjugation involution $\sigma$ on $A$. (This is the involution with $\sigma(i)=-i$ and $\sigma(j)=-j$, which is the unique symplectic involution on $A$.) Since $A$ is a division algebra, $\sigma$ must be anisotropic. The residue involution $\sigma_{0}$ is the conjugation involution on $A_{0}$, which is isotropic, since $\sigma_{0}(t) t=\operatorname{Nrd}_{A_{0}}(t)$ for any $t$ in the split quaternion algebra $A_{0}$. So, by Corollary 6.2(b) there is no $\sigma$-special $v$-gauge on $A$. For any unit $u \in A^{\times}$the map $\varphi_{u}$ defined by

$$
\varphi_{u}(x)=\varphi\left(u x u^{-1}\right) \quad \text { for } x \in A
$$


is a $v$-gauge on $A$, and Prop. 1.17 of [18] shows that $\varphi_{u}=\varphi$ if and only if $\tilde{u}$ is invertible in $\operatorname{gr}_{\varphi}(A)$, which is not a graded division ring. But, for every $u \in A^{\times}$, since $\varphi$ is invariant under $\sigma$ and $\sigma(u) u$ is central,

$$
\begin{aligned}
\varphi_{u}(\sigma(x)) & =\varphi\left(\sigma\left[\left(\sigma\left(u^{-1}\right) x \sigma(u)\right]\right)=\varphi\left(\sigma\left(u^{-1}\right) x \sigma(u)\right)\right. \\
& =\varphi_{u}\left([\sigma(u) u]^{-1} x[\sigma(u) u]\right)=\varphi_{u}(x),
\end{aligned}
$$

showing that $\varphi_{u}$ is invariant under $\sigma$.

Open Access This article is distributed under the terms of the Creative Commons Attribution Noncommercial License which permits any noncommercial use, distribution, and reproduction in any medium, provided the original author(s) and source are credited.

\section{References}

1. Bourbaki, N.: Elements of Mathematics, Commutative Algebra. Addison-Wesley, Reading (1972). English trans. of Éléments de Mathématique, Algèbre Commutative

2. Dejaiffe, I.: Somme orthogonale d'algèbres à involution et algèbre de Clifford. Comm. Algebra 26, 1589-1612 (1998)

3. Dejaiffe, I., Lewis, D.W., Tignol, J.-P.: Witt equivalence of central simple algebras with involution. Rend. Circ. Mat. Palermo 49(2), 325-342 (2000)

4. Dixmier, J.: Les $C^{*}$-algèbres et leurs représentations. Gauthier-Villars \& Cie, Éditeur, Paris (1964). English trans.: $C^{*}$-Algebras. North-Holland, Amsterdam (1977)

5. Ershov, Yu.L.: Valued division rings. In: Fifth All Union Symposium, Theory of Rings, Algebras, and Modules, pp. 53-55. Akad. Nauk SSSR Sibirsk. Otdel., Inst. Mat., Novosibirsk (1982, in Russian)

6. Ershov, Yu.L.: Henselian valuations of division rings and the group $S K_{1}$. Math. USSR Sbornik 45, 63-71 (1983)

7. Ershov, Yu.L.: Multi-Valued Fields. Kluwer, New York (2001)

8. Endler, O.: Valuation Theory. Springer, New York (1972)

9. Engler, A.J., Prestel, A.: Valued Fields. Springer, Berlin (2005)

10. Hwang, Y.-S., Wadsworth, A.R.: Algebraic extensions of graded and valued fields. Comm. Algebra 27, 821-840 (1999)

11. Hwang, Y.-S., Wadsworth, A.R.: Correspondences between valued division algebras and graded division algebras. J. Algebra 220, 73-114 (1999)

12. Knus, M.-A., Merkurjev, A.S., Rost, M., Tignol, J.-P.: The Book of Involutions. Coll. Pub. 44. Amer. Math. Soc., Providence (1998)

13. Larmour, D.W.: A Springer Theorem for Hermitian forms. Math. Z. 252, 459-472 (2006)

14. Morandi, P.: The Henselization of a valued division algebra. J. Algebra 122, 232-243 (1989)

15. Pierce, R.S.: Associative Algebras. Springer, New York (1982)

16. Renard, J.-F., Tignol, J.-P., Wadsworth, A.R.: Graded Hermitian forms and Springer's theorem. Indag. Math., N.S. 18, 97-134 (2007)

17. Schilling, O.F.G.: The Theory of Valuations. Amer. Math. Soc., New York (1950)

18. Tignol, J.-P., Wadsworth, A.R.: Value functions and associated graded rings for semisimple algebras. Trans. Am. Math. Soc. 362, 687-726 (2010)

19. Wadsworth, A.R.: Extending valuations to finite-dimensional division algebras. Proc. Am. Math. Soc. 98, 20-22 (1986)

20. Weil, A.: Algebras with involutions and the classical groups. J. Indian Math. Soc. (N.S.) 24, 589-623 (1960) 